Bulletin of the Seismological Society of America. Vol. 54, No. 2, pp. 559-570. April, 1964

\title{
STUDY OF LOVE AND RAYLEIGH WAVES FROM EARTHQUAKES WITH FAULT PLANE SOLUTIONS OR WITH KNOWN FAULTING Part 3. TABLE OF SOURCE PHASE DIFFERENCES BETWEEN RAYLEIGH AND LOVE WAVES
}

\author{
By KeIITI AKI
}

\begin{abstract}
The table of source phase differences between Rayleigh and Love waves which was described in Part 1 and used in Part 2 is presented in a concise form for the case of a surface focus.
\end{abstract}

\section{INTRODUCTION}

The source phases $\phi_{R}$ and $\phi_{L}$ of Rayleigh and Love waves as defined in equation 1 of Part 1 are computed for various fault systems on the assumption of the double couple and the modified single couple. The method of computation was described in detail in Part 1. We specify a fault motion by the following two parameters: (1) the difference between the azimuth $\varphi_{1}$ of the dip direction of the fault plane and $\varphi_{2}$ of the auxiliary plane, and (2) the dip angle $\delta_{1}$ of the fault plane. The auxiliary plane is the plane normal to the direction of the fault motion, and is usually defined in the fault plane solutions given from initial motion data. The slip angle, which is the angle between fault strike and motion direction, may be computed from the above two parameters by equations 15 and 16 of Part 1.

For a fault with the above two parameters given, four types of motion are possible: left-lateral reverse, left-lateral normal, right-lateral reverse and right-lateral normal motion as schematically illustrated in figure 1. It can be shown that if we know the value of $\phi_{R}-\phi_{L}$ for one type of motion, we can derive the values for the other three types from it.

First, the value of $\phi_{R}-\phi_{L}$ is the same at any azimuth for right-lateral reverse motion and for left-lateral normal motion. This is because the two motions are identical except for the sense. The same can be said for right-lateral normal and left-lateral reverse motion.

Secondly, the value of $\phi_{R}-\phi_{L}$ at an azimuth $\theta$ for left-lateral reverse motion may be obtained from the value at $180^{\circ}-\theta$ for right-lateral reverse motion, where $\theta$ is measured clockwise from the strike of fault as shown in figure 1 . The relation between the two values may be expressed as

$\left(\phi_{R}-\phi_{L}\right)$ at $\theta$ for left-lateral reverse motion

$$
=0.5+\left(\phi_{R}-\phi_{L}\right) \text { at } 180^{\circ}-\theta \text { for right-lateral reverse motion. }
$$

Similarly, we have the relation that,

$\left(\phi_{R}-\phi_{r}\right)$ at, $\theta$ for left-lateral normal motion

$$
=0.5+\left(\phi_{R}-\phi_{T_{1}}\right) \text { at } 180^{\circ}-\theta \text { for right-lateral normal motion. }
$$


These relations hold for the double couple model as well as for the modified single couple model.

The proof of the above relations may be obtained by decomposing the force system into a vertical component $f_{V}$, a horizontal component in the strike direction $f_{S H}$ and one in the dip direction $f_{D H}$. It can be shown that the transformations

$$
\begin{aligned}
f_{V}{ }^{\prime} & =f_{V} \\
f_{D H}^{\prime} & =f_{D H} \\
f_{S H}^{\prime} & =-f_{S H}
\end{aligned}
$$
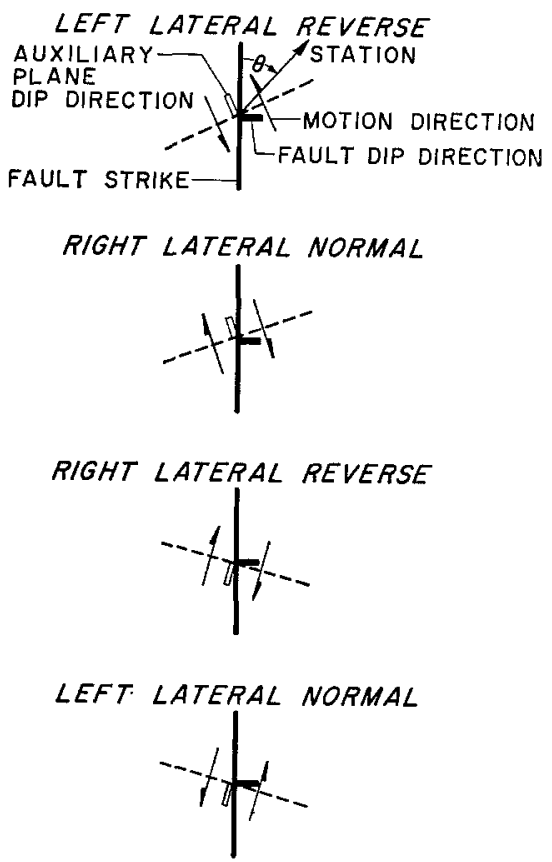

FIG. 1. Four types of motion on a fault with given two parameters; namely, dip direction difference between fault plane and auxiliary plane and dip angle of fault plane. The horizontal projection of the motion direction is indicated by pairs of arrows.

and

$$
\theta^{\prime}=180^{\circ}-\theta
$$

will not change the phase $\phi_{R}$ of Rayleigh waves but will change the phase $\phi_{L}$ of Love waves by 0.5 circle for the single couple, double couple and modified single couple. (See, e.g., equations 57 and 61 of Haskell's (1963) paper.)

Thus, it is only necessary to show the values for one type of fault motion. In our table, the values are given for left-lateral reverse motion such as shown at the top of figure 1.

Further simplification comes from the fact that for a given fault motion the value of $\phi_{R}-\phi_{L}$ at an azimuth $\theta$ and that at $\theta+180^{\circ}$ are related. The relation may be 
expressed as

$$
\left\{\left(\phi_{R}-\phi_{L}\right) \text { at } \theta\right\}+\left\{\left(\phi_{R}-\phi_{L}\right) \text { at } \theta+180^{\circ}\right\}=0
$$

This relation holds also for the single couple, double couple and modified single couple model. The proof of this relation may be obtained from the expression for displacement of Rayleigh waves as given by equation 57 (for a single couple) or equation 61 (for a double couple) of Haskell's (1963) paper. The factor including the azimuthal angle in these equations shows a complex conjugate relation between azimuths $\theta$ and $\theta+180^{\circ}$. Since the same relation holds for Love waves, we get the relation for $\phi_{R}-\phi_{L}$ shown above. Thus, it is only necessary to give the values of $\phi_{R}-\phi_{L}$ for the azimuth from 0 to $180^{\circ}$.

The following tables give the values of $\phi_{R}-\phi_{L}$ in parts of a circle for left-lateral reverse motion with the following parameters: the dip angle of the fault (designated as $D I P$ in the table) ranges from $1^{\circ}$ to $89^{\circ}$, with the interval of $8^{\circ}$; the difference in the azimuth of dip direction between the fault plane and auxiliary plane varies from $90^{\circ}$ to $180^{\circ}$ with various intervals; the slip angle (designated as SLIP). The azimuth to the station (designated as AZM) is measured clockwise from the strike direction as shown at the top of figure 1 . Note that the azimuths shown in the table lie in the dipping side of the fault.

The following tables give the values of $\phi_{R}-\phi_{L}$ corresponding to very shallow earthquakes. The depth is assumed as zero in the computation of $\phi_{R}$, and the depth parameter for Love waves defined in Part 1 is taken as 0.1 .

\section{EXAMPLES}

We shall show how to obtain the value of $\phi_{R}-\phi_{L}$ for a given fault motion from the tables by using the Kern County main shock and shock M3 of Part 2 as examples.

As described in Part 2, the fault motion associated with the Kern County earthquake is left-lateral reverse on a fault with strike direction $\mathrm{N} 50^{\circ} \mathrm{E}$ and dip angle $63^{\circ}$. The fault dips toward south-east and the difference between the azimuth of dip direction of the fault plane and that of the auxiliary plane is $140^{\circ}$.

The azimuths to the European stations (table 6 of Part 2 ) are about $\mathrm{N} 30^{\circ} \mathrm{E}$ from the epicenter, and are about $340^{\circ}$ if measured from the fault strike in the way as shown in figure 1.

First, we look for the value of $\phi_{R}-\phi_{L}$ at the azimuth of $160^{\circ}\left(=340^{\circ}-180^{\circ}\right)$ for the dip angle of $63^{\circ}$ and the dip direction difference of $140^{\circ}$. We find from Table 10 that the corresponding value is -0.167 and -0.019 for the modified single couple and the double couple respectively. Then, from the conjugate relation described before, we get the value of 0.167 and 0.019 for the azimuth $340^{\circ}$ for the two models.

The next example is shock M3, for which the fault parameters are given in table 2 of Part 2. The type of fault motion of this shock is illustrated in figure 2. If plane $a$ is the actual fault, the motion is right-lateral normal, and if plane $b$ is the actual fault, the motion is left-lateral normal.

As mentioned before, right-lateral normal motion gives the same value of $\phi_{R}-$ $\phi_{L}$ as left-lateral reverse motion for which the tables show the values. The dip angle of plane $a$ is $18^{\circ}$, the dip direction difference is $132^{\circ}$, and the azimuth to Pasadena 
Left lateral Reverse fault, dip direction difference $=90.0$

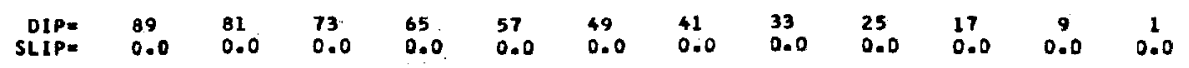

$12 M$
10
20
30
40
50
60
70
80
90
100
110
120
130
140
150
160
170
180
424
10
20
30
40
50
60
70
80
90
100
110
120
130
140
150
160
170
180

MODIFIED SINGLE COUPLE

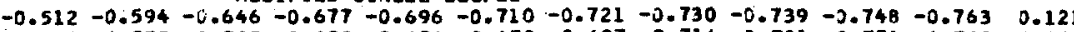
$\begin{array}{llllllllllll}-0.506 & -0.553 & -0.5595 & -0.628 & -0.656 & -0.678 & -0.697 & -0.714 & -0.731 & -0.751 & -0.782 & 0.061\end{array}$

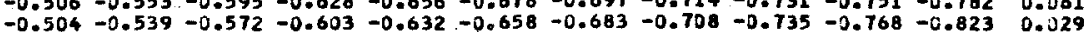

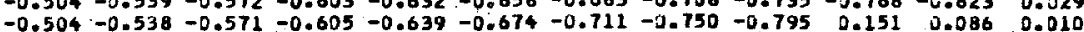

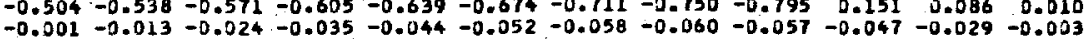
$\begin{array}{lllllllllllll}-0.002 & -0.017 & -0.032 & -0.047 & -0.062 & -0.077 & -0.092 & -0.104 & -0.113 & -0.113 & -0.088 & -0.013\end{array}$ $\begin{array}{llllllllllllll}-0.002 & -0.016 & -0.031 & -0.046 & -0.0662 & -0.078 & -0.094 & -0.110 & -0.123 & -0.130 & -0.113 & -0.019\end{array}$ $\begin{array}{llllllllllll}-0.002 & -0.016 & -0.030 & -0.045 & -0.061 & -0.077 & -0.094 & -0.110 & -0.126 & -0.135 & -0.124 & -0.023\end{array}$ $\begin{array}{llllllllllll}0.257 & 0.281 & 0.299 & 0.315 & 0.326 & 0.334 & 0.337 & 0.338 & 0.338 & 0.341 & 0.362 & 0.475\end{array}$ $0.498 \quad 0.484 \quad 0.4690 .454 \quad 0.4390 .4230 .406 \quad 0.3890 .3740 .364 \quad 0.376 \quad 0.477$ $\begin{array}{llllllllllll}0.498 & 0.484 & 0.469 & 0.454 & 0.439 & 0.423 & 0.406 & 0.399 & 0.374 & 0.364 & 0.376 & 0.477\end{array}$ $\begin{array}{llllllllllll}0.498 & 0.483 & 0.469 & 0.453 & 0.438 & 0.422 & 0.406 & 0.390 & 0.377 & 0.370 & 0.387 & 0.481 \\ 0.498 & 0.483 & 0.468 & 0.453 & 0.438 & 0.423 & 0.408 & 0.396 & 0.387 & 0.387 & 0.412 & 0.487\end{array}$ $\begin{array}{llllllllllll}0.498 & 0.483 & 0.468 & 0.453 & 0.438 & 0.423 & 0.408 & 0.396 & 0.387 & 0.387 & 0.412 & 0.487 \\ 0.499 & 0.487 & 0.476 & 0.465 & 0.456 & 0.448 & 0.442 & 0.440 & 0.443 & 0.453 & 0.471 & 0.497\end{array}$ $\begin{array}{llllllllllll}-0.004 & -0.038 & -0.071 & -0.105 & -0.239 & -0.174 & -0.210 & -0.250 & -0.295 & 0.652 & 0.586 & 0.510\end{array}$

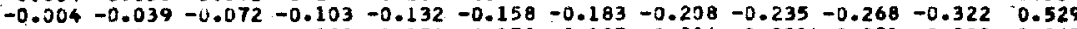
$\begin{array}{lllllllllllll}-0.006 & -0.053 & -0.095 & -0.128 & -0.156 & -0.178 & -0.197 & -0.214 & -0.231 & -0.251 & -0.282 & 0.561\end{array}$ $\begin{array}{lllllllllllll}-0.012 & -0.094 & -0.146 & -0.177 & -0.196 & -0.210 & -0.221 & -0.230 & -0.239 & -0.248 & -0.263 & 0.621\end{array}$

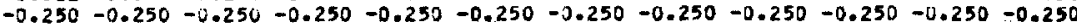
DOUBLE COUPLE

$\begin{array}{llllllllllll}-0.500 & -0.5 & -0.501 & -0.501 & -0.502 & -0.533 & -0.503 & -0.505 & -0.506 & -0.510 & -0.519 & 0.368\end{array}$

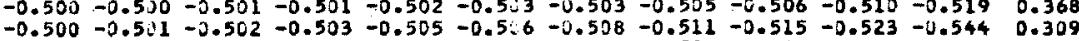

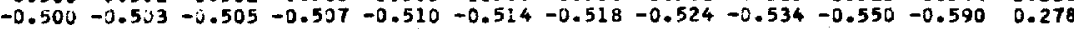
$\begin{array}{llllllllllllll}-0.501 & -0.539 & -0.518 & -0.527 & -0.538 & -0.550 & -0.564 & -0.582 & -0.607 & 0.360 & 0.314 & 0.258\end{array}$

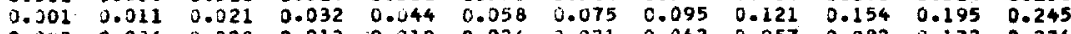
$\begin{array}{llllllllllll}0.000 & 0.034 & 0.008 & 0.013 & 0.018 & 0.024 & 0.031 & 0.042 & 0.057 & 0.082 & 0.132 & 0.236\end{array}$ $\begin{array}{lllllllllllll}0.300 & 0.0 j 3 & 0.006 & 0.009 & 0.013 & 0.017 & 0.022 & 0.030 & 0.041 & 0.061 & 0.105 & 0.230\end{array}$ $\begin{array}{llllllllllll}0.000 & 0.033 & 0.005 & 0.008 & 0.011 & 0.015 & 0.019 & 0.026 & 0.035 & 0.053 & 0.094 & 0.230\end{array}$ $\begin{array}{llllllllllll}0.503 & 0.033 & 1.005 & 0.008 & 0.011 & 0.015 & 0.019 & 0.026 & 0.035 & 0.053 & 0.094 & 0.230 \\ 0.282 & 0.287 & 0.305 & 0.320 & 0.331 & 0.338 & 0.341 & 0.342 & 0.341 & 0.343 & 0.364 & 0.475\end{array}$ $\begin{array}{llllllllllll}0.282 & 0.287 & 0.305 & 0.320 & 0.331 & 0.338 & 0.341 & 0.342 & 0.341 & 0.343 & 0.364 & 0.475 \\ 0.500 & 0.5 j 3 & 0.505 & 0.508 & 0.511 & 0.514 & 0.519 & 0.525 & 0.535 & 0.552 & 0.592 & 0.717\end{array}$ $\begin{array}{lllllllllllll}0.500 & 0.5 j 3 & 0.506 & 0.509 & 0.513 & 0.517 & 0.522 & 0.530 & 0.541 & 0.560 & 0.604 & 0.724\end{array}$

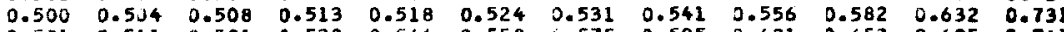

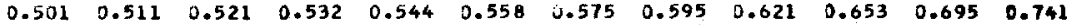

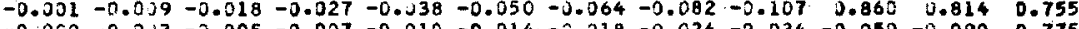

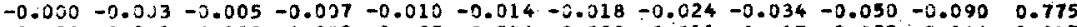
$\begin{array}{lllllllllllll}-0.000 & -0.0 j 1 & -0.002 & -0.003 & -0.005 & -0.036 & -0.008 & -0.011 & -0.015 & -0.023 & -0.0 .044 & 0.837\end{array}$ $-0.000-0.030-0.101-0.031-0.002-0.0033-0.003-0.005-0.006-0.010-0.019 \quad 0.866$ $\begin{array}{llllllllllll}-0.372 & -0.264 & -0.228 & -0.214 & -0.208 & -0.266 & -0.207 & -0.211 & -0.215 & -0.221 & -0.228 & -0.235\end{array}$ TABLE 1

LEFT Lateral Reverse fault, DIP DIRECTION DIFFERENCE $=91.0$

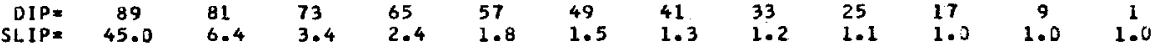

MODIFIED SINGLE COUPLE

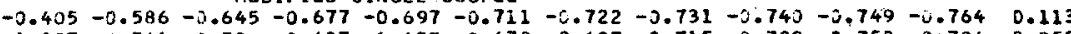
$\begin{array}{lllllllllllll}-0.397 & -0.541 & -0.590 & -0.627 & -0.655 & -0.678 & -0.697 & -0.715 & -0.732 & -0.752 & -0.784 & 0.058\end{array}$

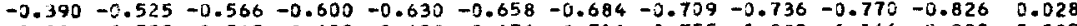
$\begin{array}{llllllllllll}-0.380 & -0.522 & -0.565 & -0.602 & -0.639 & -0.676 & -0.714 & -0.755 & -0.800 & 0.146 & 0.082 & 0.009\end{array}$

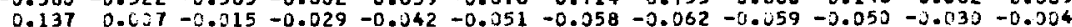
$0.1560 .039-0.019-0.039-0.057-0.074-0.089=0.103-0.113-0.113-0.089-0.013$ 0.156 . $0.023-0.011-0.034-0.053-0.072-0.089-0.107-0.122-0.129-0.113-0.019$

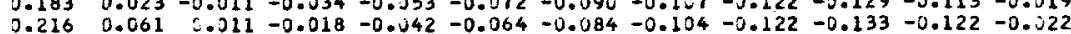

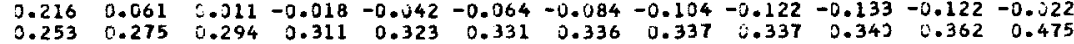

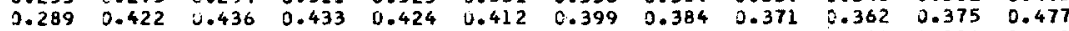
$\begin{array}{llllllllllll}0.321 & 0.449 & 0.451 & 0.442 & 0.430 & 0.417 & 0.402 & 0.388 & 0.375 & 0.369 & 0.386 & 0.481\end{array}$ $\begin{array}{llllllllllll}0.346 & 0.459 & 0.456 & 0.445 & 0.433 & 0.419 & 0.406 & 0.394 & 0.386 & 0.387 & 0.412 & 0.487\end{array}$

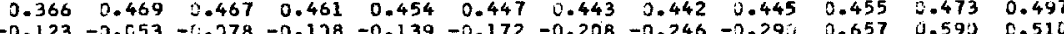

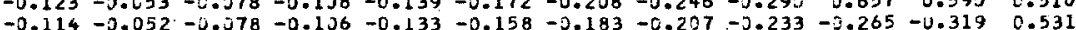

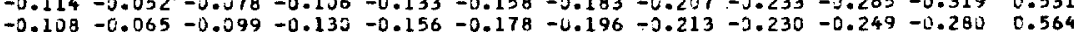

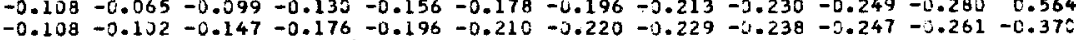
$-0.215-0.246-.248-0.249-0.249-0.249-0.249-0.249-0.249-0.249-0.248-0.234$

DOUBLE COUPLE

$-0.483-0.499-0.500-0.501-0.502-0.503-0.504-0.505-0.507-0.511-0.521 \quad 0.359$

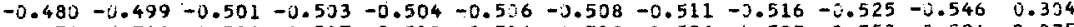

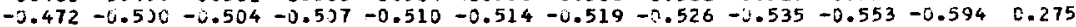

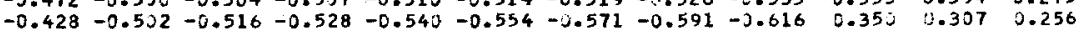

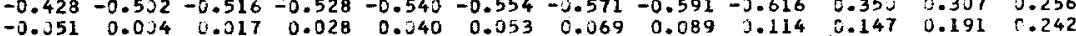
$\begin{array}{llllllllllll}-0.051 & 0.034 & 0.017 & 0.028 & 0.040 & 0.053 & 0.069 & 0.089 & 0.114 & 0.147 & 0.191 & 0.242 \\ -0.015 & 0.333 & 0.007 & 0.012 & 0.017 & 0.023 & 0.036 & 0.041 & 0.055 & 0.081 & 1.130 & 0.233\end{array}$ $\begin{array}{llllllllllll}-0.015 & 0.033 & 0.007 & 0.012 & 0.017 & 0.023 & 0.036 & 0.041 & 0.055 & 0.081 & 1.130 & 0.233 \\ -0.007 & 0.0 .52 & 0.006 & 0.009 & 0.012 & 0.017 & 0.022 & 0.029 & 0.041 & 0.060 & 4.104 & 0.227\end{array}$ $\begin{array}{llllllllllll}-0.003 & 0.032 & 0.005 & 0.008 & 0.011 & 0.014 & 0.019 & 0.025 & 0.035 & 0.052 & 0.093 & 0.223\end{array}$ $\begin{array}{lllllllllllll}0.501 & 0.5 & 0.53 & 0.505 & 0.507 & 0.510 & 0.514 & 0.518 & 0.524 & 0.534 & 0.553 & 0.590 & 0.722\end{array}$ $\begin{array}{lllllllllllll}0.503 & 0.533 & 0.505 & 0.508 & 0.511 & 0.515 & 0.519 & 0.526 & 0.535 & 0.553 & 0.593 & 0.723\end{array}$ $\begin{array}{lllllllllllll}0.508 & 0.524 & 0.506 & 0.539 & 0.513 & 0.517 & 0.523 & 0.530 & 0.541 & 0.561 & 0.635 & 0.727\end{array}$ $\begin{array}{llllllllllll}0.517 & 0.516 & 0.510 & 0.514 & 0.519 & 0.525 & 0.532 & 0.542 & 0.558 & 0.583 & 0.634 & 0.734\end{array}$

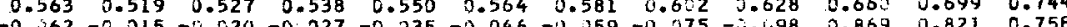

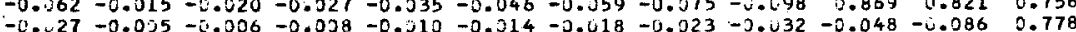

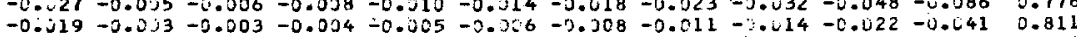
$-0.017-0.042-0.002-0.002-0.002-0.0 .3-0.003-0.004-0.006-0.069-0.017-0.124$ $-0.216,-0.002-0.001-0.001-0.002-0.0 .2-0.002-0.003-0.003-0.005-0.009-0.068$ 
LEFT LATERAL REVERSE FAULT, dip DIRECTION DIFFERENCE = 92.0

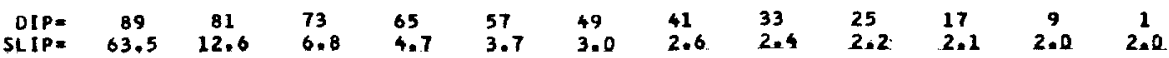

MODIFIEO SIMGLE COUPLE

\begin{tabular}{|c|c|c|c|c|c|c|c|c|c|c|c|c|}
\hline $\begin{array}{r}A Z M \\
10 \\
20 \\
30 \\
40 \\
50 \\
60 \\
70 \\
80 \\
90 \\
100 \\
110 \\
120 \\
130 \\
140\end{array}$ & $\begin{array}{r}-0.345 \\
-0.341 \\
-0.334 \\
-0.327 \\
0.187 \\
0.200 \\
0.216 \\
0.234 \\
0.253 \\
0.271 \\
0.289 \\
0.305 \\
0.318 \\
-0.173 \\
-0.165 \\
-0.160 \\
-0.157 \\
-0.182\end{array}$ & $\begin{array}{r}-0.577 \\
-0.528 \\
-0.539 \\
-0.535 \\
0.028 \\
0.037 \\
0.062 \\
0.125 \\
0.275 \\
0.382 \\
0.421 \\
0.438 \\
0.452 \\
-0.067 \\
-0.065 \\
-0.075 \\
-0.109 \\
-0.242\end{array}$ & $\begin{array}{r}-0.643 \\
-0.585 \\
-0.559 \\
-0.558 \\
-0.005 \\
-0.004 \\
0.011 \\
0.058 \\
0.294 \\
0.411 \\
0.435 \\
0.445 \\
0.459 \\
-0.084 \\
-0.083 \\
-0.103 \\
-0.148 \\
-0.246\end{array}$ & $\begin{array}{l}-0.625 \\
-0.597 \\
-0.600 \\
-0.024 \\
-0.030 \\
-0.019 \\
0.015 \\
0.311 \\
0.416 \\
0.432 \\
0.438 \\
0.457 \\
-0.110 \\
-0.109 \\
-0.132 \\
-0.176 \\
-0.247\end{array}$ & $\begin{array}{r}-0.698 \\
-0.655 \\
-0.629 \\
-0.639 \\
-0.039 \\
-0.051 \\
-0.043 \\
-0.018 \\
0.323 \\
0.412 \\
0.423 \\
0.428 \\
0.452 \\
-0.140 \\
-0.134 \\
-0.156 \\
-0.195 \\
-0.248\end{array}$ & $\begin{array}{r}\text { CUUPL } \\
-0.712 \\
-0.678 \\
-0.657 \\
-0.678 \\
-0.050 \\
-0.070 \\
-0.065 \\
-0.247 \\
0.331 \\
0.404 \\
0.412 \\
0.416 \\
0.447 \\
-0.171 \\
-0.159 \\
-0.178 \\
-0.209 \\
-0.248\end{array}$ & $\begin{array}{r}-0.723 \\
-0.698 \\
-0.684 \\
-0.717 \\
-0.059 \\
-0.087 \\
-0.085 \\
-0.072 \\
0.336 \\
0.393 \\
0.399 \\
0.404 \\
0.444 \\
-0.205 \\
-0.182 \\
-0.196 \\
-0.220 \\
-0.248\end{array}$ & $\begin{array}{l}-0.732 \\
-0.716 \\
-0.710 \\
-0.759 \\
-0.063 \\
-0.101 \\
-0.104 \\
-0.096 \\
0.337 \\
0.380 \\
0.385 \\
0.393 \\
0.443 \\
-0.242 \\
-0.256 \\
-0.213 \\
-0.229 \\
-0.248\end{array}$ & $\begin{array}{r}-0.741 \\
-0.733 \\
-0.738 \\
-0.006 \\
-0.061 \\
-0.112 \\
-0.120 \\
-0.116 \\
0.337 \\
0.368 \\
0.373 \\
0.385 \\
0.448 \\
-0.285 \\
-0.232 \\
-0.229 \\
-0.237 \\
-0.248\end{array}$ & $\begin{array}{r}-0.750 \\
-0.753 \\
-0.772 \\
0.141 \\
-0.052 \\
-0.112 \\
-0.128 \\
-0.130 \\
0.340 \\
0.361 \\
0.368 \\
0.387 \\
0.458 \\
0.662 \\
-0.263 \\
-0.248 \\
-0.246 \\
-0.248\end{array}$ & $\begin{array}{r}-0.766 \\
-0.787 \\
-0.829 \\
0.078 \\
-0.032 \\
-0.089 \\
-0.112 \\
-0.121 \\
0.362 \\
0.374 \\
0.386 \\
0.412 \\
0.475 \\
0.594 \\
-0.316 \\
-0.277 \\
-0.259 \\
-0.246\end{array}$ & $\begin{array}{r}0.106 \\
0.055 \\
0.027 \\
0.009 \\
-0.004 \\
-0.013 \\
-0.019 \\
-0.022 \\
0.475 \\
0.477 \\
0.481 \\
0.487 \\
0.497 \\
0.511 \\
0.532 \\
0.567 \\
-0.361 \\
-0.219\end{array}$ \\
\hline $\begin{array}{r}\text { ALM } \\
10 \\
20 \\
30 \\
40 \\
50 \\
60 \\
70 \\
80 \\
90 \\
100 \\
110 \\
120 \\
130 \\
140 \\
150 \\
160 \\
170 \\
180\end{array}$ & $\begin{array}{l}-0.467 \\
-0.461 \\
-0.444 \\
-0.368 \\
-0.387 \\
-0.029 \\
-0.014 \\
-0.006 \\
0.501 \\
0.506 \\
0.515 \\
0.534 \\
0.620 \\
-0.102 \\
-0.050 \\
-0.037 \\
-0.033 \\
-0.032\end{array}$ & $\begin{array}{r}-0.497 \\
-0.497 \\
-0.496 \\
-0.493 \\
-0.001 \\
0.001 \\
0.002 \\
0.002 \\
0.503 \\
0.503 \\
0.505 \\
0.508 \\
0.529 \\
-0.020 \\
-0.068 \\
-0.005 \\
-0.004 \\
-0.004\end{array}$ & $\begin{array}{r}-0.499 \\
-0.500 \\
-0.502 \\
-0.513 \\
0.013 \\
0.006 \\
0.005 \\
0.005 \\
0.505 \\
0.505 \\
0.507 \\
0.511 \\
0.534 \\
-0.021 \\
-0.007 \\
-0.004 \\
-0.003 \\
-0.002\end{array}$ & $\begin{array}{l}\text { DOUBLE } \\
-0.500 \\
-0.502 \\
-0.506 \\
-0.529 \\
0.024 \\
0.011 \\
0.008 \\
0.007 \\
0.507 \\
0.508 \\
0.510 \\
0.515 \\
0.544 \\
-0.026 \\
-0.009 \\
-0.004 \\
-0.002 \\
-0.001\end{array}$ & $\begin{array}{r}-0.501 \\
-0.504 \\
-0.510 \\
-0.544 \\
0.036 \\
0.016 \\
0.012 \\
0.011 \\
0.510 \\
0.511 \\
0.513 \\
0.519 \\
0.557 \\
-0.033 \\
-0.011 \\
-0.005 \\
-0.002 \\
-0.001\end{array}$ & $\begin{array}{r}-0.562 \\
-0.566 \\
-0.514 \\
-0.560 \\
0.049 \\
0.022 \\
0.016 \\
0.014 \\
0.514 \\
0.515 \\
0.517 \\
0.525 \\
0.572 \\
-0.042 \\
-0.013 \\
-0.056 \\
-0.003 \\
-0.001\end{array}$ & $\begin{array}{r}-0.504 \\
-0.509 \\
-0.520 \\
-0.578 \\
0.064 \\
0.030 \\
0.022 \\
0.019 \\
0.518 \\
0.519 \\
0.523 \\
0.533 \\
0.589 \\
-0.054 \\
-0.017 \\
-0.008 \\
-0.003 \\
-0.001\end{array}$ & $\begin{array}{r}-0.505 \\
-0.512 \\
-0.527 \\
-0.600 \\
0.083 \\
0.040 \\
0.029 \\
0.025 \\
0.524 \\
0.526 \\
0.530 \\
0.544 \\
0.611 \\
-0.069 \\
-0.022 \\
-0.010 \\
-0.004 \\
-0.001\end{array}$ & $\begin{array}{r}-0.508 \\
-0.517 \\
-0.537 \\
0.373 \\
0.108 \\
0.054 \\
0.040 \\
0.435 \\
0.534 \\
0.535 \\
0.542 \\
0.559 \\
0.636 \\
-0.091 \\
-0.030 \\
-0.014 \\
-0.005 \\
-0.001\end{array}$ & $\begin{array}{r}-0.512 \\
-0.526 \\
-0.556 \\
0.340 \\
0.142 \\
0.079 \\
0.060 \\
0.052 \\
0.550 \\
0.553 \\
0.562 \\
0.585 \\
0.667 \\
-0.123 \\
-0.045 \\
-0.021 \\
-0.008 \\
-0.001\end{array}$ & $\begin{array}{r}-0.523 \\
-0.549 \\
-0.599 \\
0.301 \\
0.187 \\
0.129 \\
0.104 \\
0.092 \\
0.590 \\
0.593 \\
0.606 \\
0.635 \\
0.704 \\
0.828 \\
-0.081 \\
-0.039 \\
-0.015 \\
-0.002\end{array}$ & $\begin{array}{l}0.352 \\
0.301 \\
0.273 \\
0.255 \\
0.242 \\
0.233 \\
0.227 \\
0.223 \\
0.722 \\
0.723 \\
0.727 \\
0.734 \\
0.744 \\
0.759 \\
0.780 \\
0.815 \\
-0.114 \\
-0.015\end{array}$ \\
\hline
\end{tabular}

TABLE 3

LEFT LATERAL REVERSE FAULT, DIP OIRECTION DIFFERENCE $=\mathbf{9 3 . 0}$

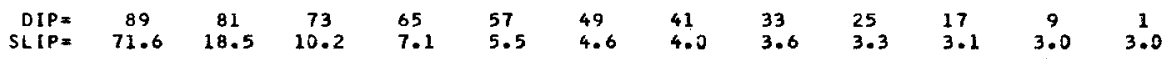

MODIFIED SINGLE COUPLE

$\begin{array}{llllllllllll}-0.316 & -0.567 & -0.642 & -0.677 & -0.698 & -0.712 & -0.723 & -0.733 & -0.742 & -0.752 & -0.768 & 0.100\end{array}$ $\begin{array}{llllllllllll}-0.313 & -0.514 & -0.580 & -0.623 & -0.0 .654 & -0.678 & -0.698 & -0.716 & -0.734 & -0.755 & -0.789 & 0.053\end{array}$ $\begin{array}{lllllllllllll}-0.309 & -0.494 & -0.552 & -0.593 & -0.627 & -0.657 & -0.684 & -0.711 & -0.739 & -0.774 & -0.832 & 0.026\end{array}$ $\begin{array}{lllllllllllll}-0.303 & -0.488 & -0.550 & -0.597 & -0.639 & -0.680 & -0.721 & -0.764 & 0.0 .812 & 0.135 & 0.075 & 0.008\end{array}$ $0.208 \quad 0.049 \quad 0.006-0.018-0.335-0.349-0.059-0.064-0.063-0.054-0.033-0.004$

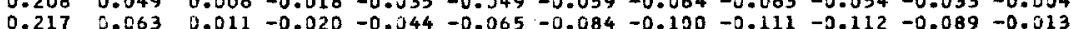

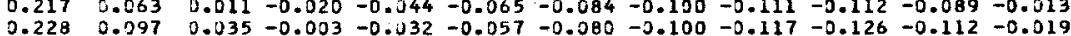

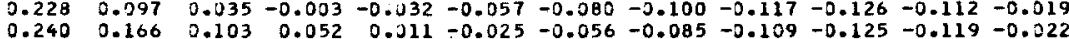

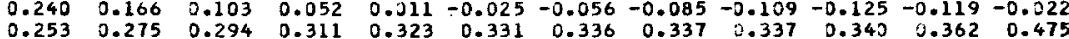
$\begin{array}{llllllllllll}0.265 & 0.357 & 0.393 & 0.403 & 0.403 & 0.397 & 0.387 & 0.376 & 0.365 & 0.359 & 0.374 & 0.477\end{array}$ $\begin{array}{llllllllllll}0.277 & 0.398 & 0.422 & 0.423 & 0.417 & 0.407 & 0.395 & 0.383 & 0.372 & 0.368 & 0.386 & 0.481\end{array}$ $\begin{array}{llllllllllll}0.288 & 0.419 & 0.435 & 0.432 & 0.424 & 0.413 & 0.402 & 0.392 & 0.385 & 0.387 & 0.412 & 0.487\end{array}$

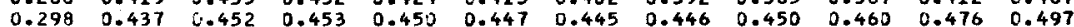

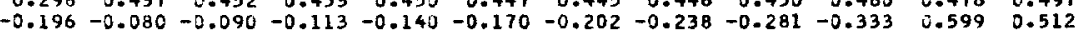
$\begin{array}{lllllllll}-0.189-0.076-0.089-0.111-0.135 & -0.159-0.182 & -0.238 & -0.281 & -0.333 & 0.599 & 0.512\end{array}$ $\begin{array}{llllllllllll}-0.189 & -0.076 & -0.089 & -0.111 & -0.135 & -0.159 & -0.182 & -0.205 & -0.230 & -0.261 & -0.313 & 0.533 \\ -0.185 & -0.085 & -0.107 & -0.133 & -0.157 & -0.177 & -0.195 & -0.212 & -0.228 & -0.247 & -0.275 & 0.571\end{array}$

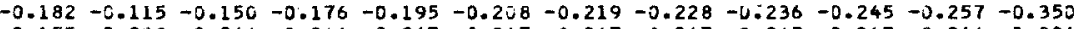

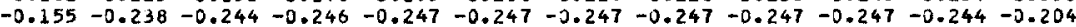
DOUBLE COUPLE

$\begin{array}{llllllllllll}-0.451 & -0.495 & -0.498 & -0.500 & -0.501 & -0.502 & -0.504 & -0.506 & -0.508 & -0.513 & -0.525 & 0.345\end{array}$ $\begin{array}{llllllllllll}-0.442 & -0.495 & -0.499 & -0.502 & -0.504 & -0.5 j 6 & -0.509 & -0.512 & -0.518 & -0.527 & -0.552 & 0.299\end{array}$ $-0.418-0.493-0.501-0.506-0.510-0.515-0.520-0.528-0.539-0.559-0.8030 .272$

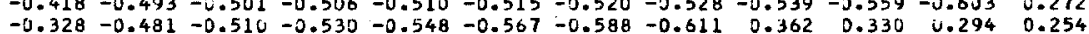

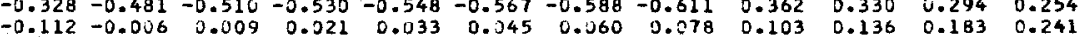
$\begin{array}{llllllllllll}-0.112 & -0.006 & 0.009 & 0.021 & 0.033 & 0.045 & 0.060 & 0.078 & 0.103 & 0.136 & 0.183 & 0.241 \\ -0.142 & -0.001 & 0.005 & 0.010 & 0.016 & 0.022 & 0.029 & 0.039 & 0.053 & 0.078 & 0.127 & 0.232\end{array}$ $\begin{array}{llllllllllll}-0.020 & 0.031 & 0.005 & 0.008 & 0.012 & 0.016 & 0.021 & 0.029 & 0.040 & 0.059 & 0.103 & 0.226\end{array}$ $\begin{array}{llllllllllll}-0.099 & 0.0 .2 & 0.005 & 0.007 & 0.010 & 0.014 & 0.019 & 0.025 & 0.035 & 0.052 & 0.092 & 0.223\end{array}$ $\begin{array}{llllllllllll}0.501 & 0.5 .33 & 0.505 & 0.507 & 0.510 & 0.514 & 0.518 & 0.524 & 0.534 & 0.553 & 0.590 & 0.722\end{array}$ $\begin{array}{llllllllllll}0.509 & 0.524 & 0.506 & 0.508 & 0.511 & 0.515 & 0.519 & 0.526 & 0.536 & 0.553 & 1.594 & 0.723\end{array}$

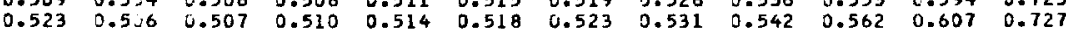

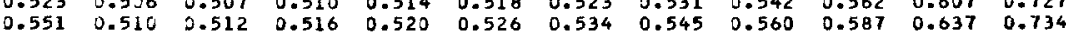

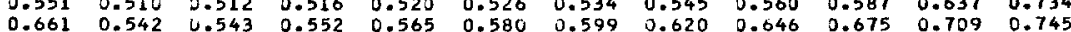

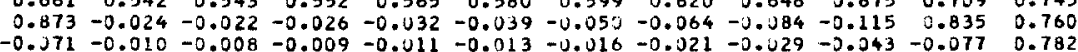

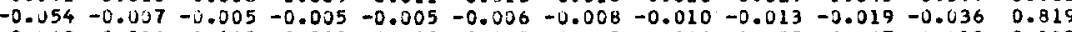
$-0.046-0.006-0.303-0.003-0.003-0.003-0.003-0.034-0.005-0.007-0.013-0.123$ $\begin{array}{lllllllllllll}-0.047 & -0.005 & -0.003 & -0.002 & -0.001 & -0.031 & -0.000 & -0.000 & 0.000 & 0.001 & 0.002 & 0.015\end{array}$ 
Left Lateral REverse fault, dip direction difference $=95.0$

$\begin{array}{rcccccccccccc}\text { DIP } & 89 & 81 & 73 & 65 & 57 & 49 & 41 & 33 & 25 & 17 & 9 & 1 \\ \text { SLIP } & 78.7 & 29.2 & 16.7 & 11.7 & 9.1 & 7.6 & 6.6 & 6.0 & 5.5 & 5.2 & 5.1 & 5.0\end{array}$

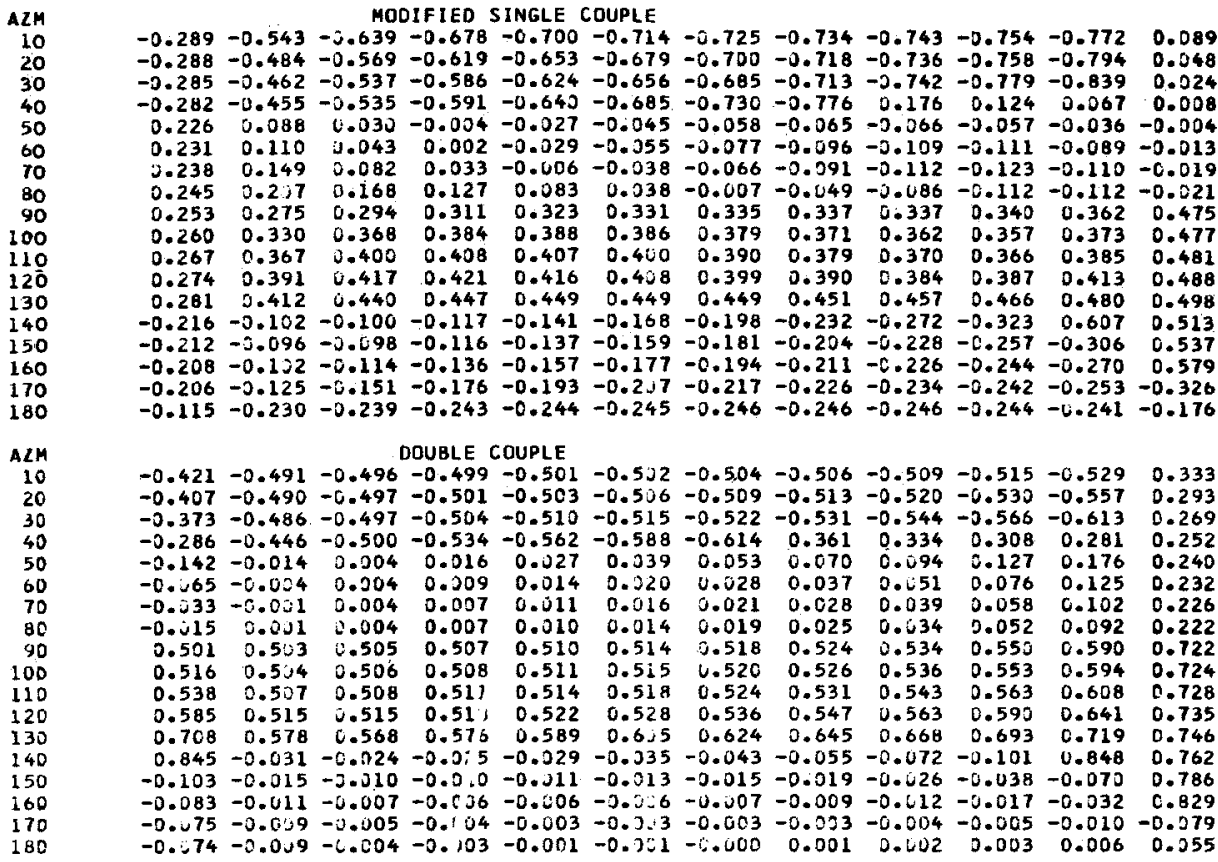
TABLE 5

LEFT LATERAL REVERSE FAULT, DIP DIRECTION DIFFERENCE $=100.0$

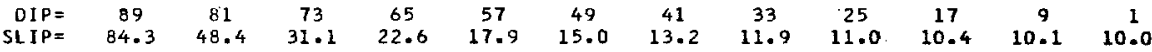

$A 2 M$
10
20
30
40
50
60
70
80
90
100
110
120
130
140
150
160
170
180
$11 M$
120
10
20
30
40
50
60
10
80
90
100
110
120
130
140
150
160
170
180

MODIFIED SINGLE CDUPLE

$\begin{array}{lllllllllllll}-0.267 & -0.466 & -0.628 & -0.679 & -0.703 & -0.718 & -0.729 & -0.739 & -0.748 & -0.760 & -0.783 & 0.069\end{array}$ $\begin{array}{lllllllllllll}-0.268 & -0.412 & -0.533 & -0.606 & -0.650 & -0.680 & -0.702 & -0.722 & -0.742 & -0.765 & -0.80 & 0.75 & 0.039\end{array}$ $\begin{array}{lllllllllllll}-0.267 & -0.393 & -0.492 & -0.563 & -0.614 & -0.554 & -0.0 .688 & -0.719 & -0.752 & -0.792 & -0.856 & 0.020\end{array}$

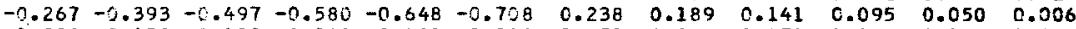

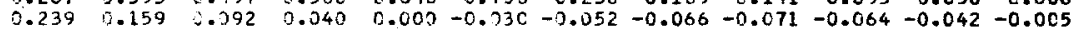

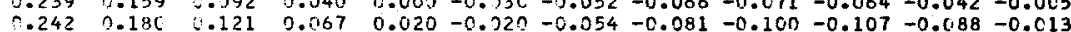

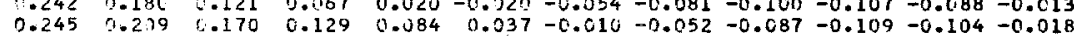

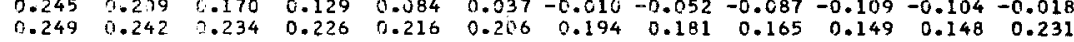

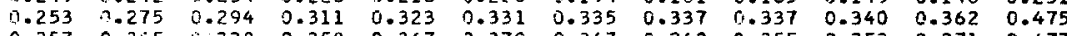
$\begin{array}{llllllllllll}0.257 & 0.365 & 0.338 & 0.358 & 0.367 & 0.370 & 0.367 & 0.362 & 0.355 & 0.353 & 0.371 & 0.477\end{array}$ $\begin{array}{llllllllllll}0.260 & 0.329 & 0.367 & 0.383 & 0.388 & 0.386 & 0.380 & 0.372 & 0.365 & 0.364 & 0.384 & 0.481 \\ 0.264 & 0.349 & 0.386 & 0.399 & 0.401 & 0.398 & 0.392 & 0.386 & 0.382 & 0.387 & 0.414 & 0.488\end{array}$

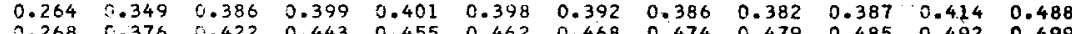
$-0.232-0.139-0.121-0.127-0.143-0.165-0.190-0.219-0.254-0.300-0.6300 .517$ $-0.230-0.132-0.118-0.126-0.142-0.160-0.180-0.200-0.222-0.248-0.2910 .547$ $-0.228-0.133-5.18-0.126-0.142-0.160-0.180-0.200-0.222-0.248-0.291 \quad 0.547$

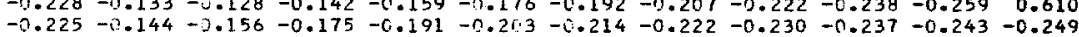
$-0.065-0.216-0.228-0.235-0.238-0.243-0.241-0.242-0.241-0.239-0.231-0.124$

DOUBLE COUPLE

$\begin{array}{lllllllllllll}-0.365 & -0.481 & -0.492 & -0.496 & -0.499 & -0.502 & -0.505 & -0.508 & -0.512 & -0.520 & -0.539 & 0.311\end{array}$ $\begin{array}{llllllllllll}-0.346 & -0.477 & -0.491 & -0.497 & -0.502 & -0.506 & -0.511 & -0.516 & -0.525 & -0.539 & -0.572 & 0.283\end{array}$ $\begin{array}{llllllllllll}-0.310 & -0.461 & -0.0 .486 & -0.499 & -0.509 & -0.518 & -0.528 & -0.541 & -0.558 & -0.586 & 0.362 & 0.263\end{array}$

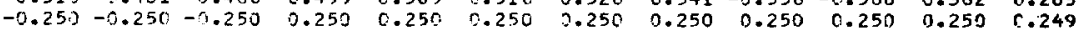

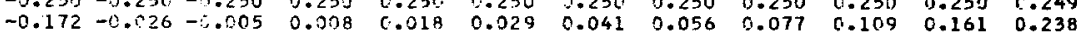

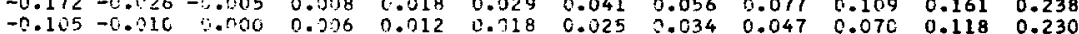

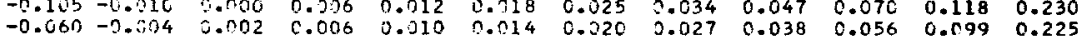
$\begin{array}{rrrrrrrrrrrr}-0.060 & -0.004 & 0.002 & 0.006 & 0.010 & 0.014 & 0.020 & 0.027 & 0.038 & 0.056 & 0.099 & 0.225 \\ 0.474 & 0.500 & 0.503 & 3.577 & 0.510 & 0.513 & 0.518 & 0.524 & 0.534 & 0.551 & 0.591 & 0.723\end{array}$

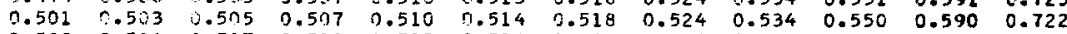
$\begin{array}{lllllllllllll}0.532 & 0.536 & 0.567 & 0.579 & 0.512 & 0.516 & 0.520 & 0.527 & 0.537 & 0.554 & 0.596 & 0.724\end{array}$

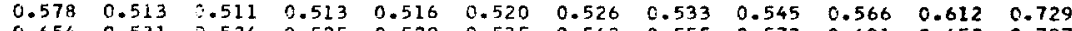
$\begin{array}{llllllllllll}0.654 & 0.531 & 0.524 & 0.525 & 0.529 & 0.535 & 0.543 & 0.555 & 0.573 & 0.601 & 0.652 & 0.737 \\ 0.75 & 0.750 & 0.750 & 0.750 & 0.750 & 0.750 & 0.750 & 0.750 & 0.750 & 0.750 & 0.750 & 0.749\end{array}$ $0.717-0.042-0.027 \quad 0.7500 .750 .0 .7500 .749$

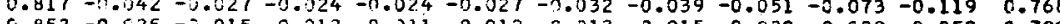
$0.852-0.025-.015-0.012-0.011-0.012-5.013-0.015-0.020-0.029-0.0520 .799$

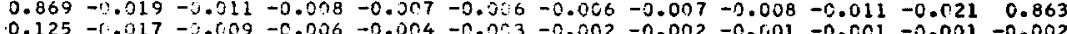

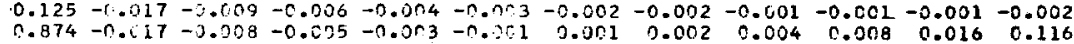
TABLE 6 
LEFT LATERAL REVERSE FAULT, DIP DIRECTION DIFFERENCE = 110.0

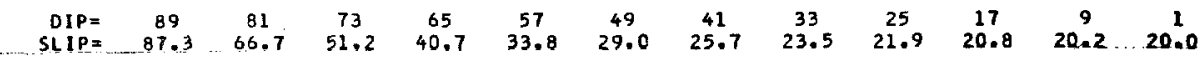

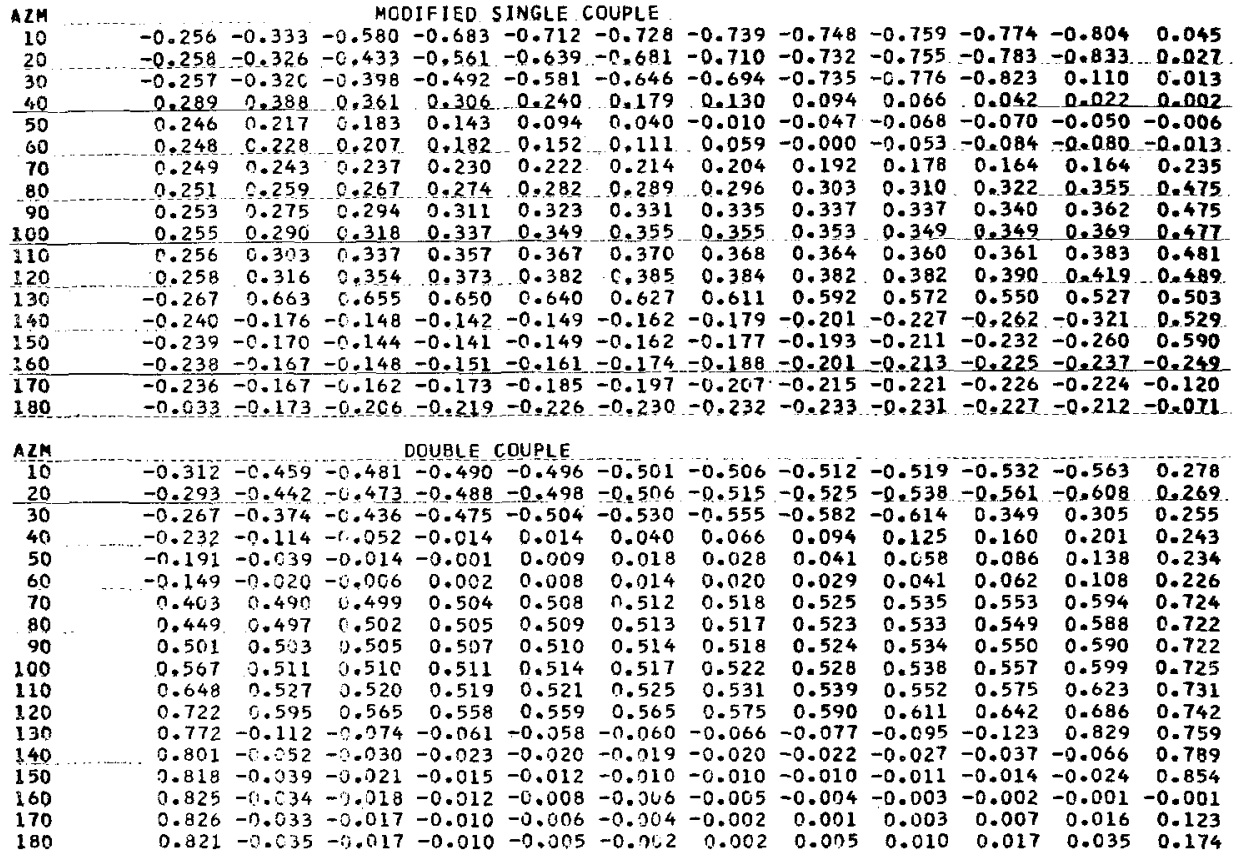

TABLE 7

LEFt lateral Reverse fault, DIP direction DIFFERENCE $=120.0$

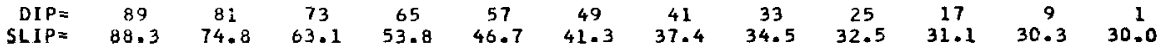

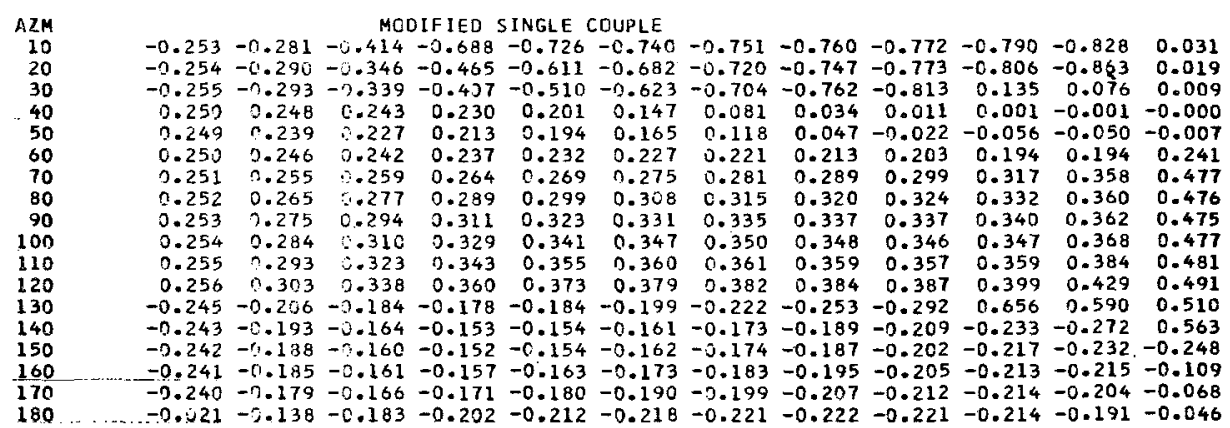

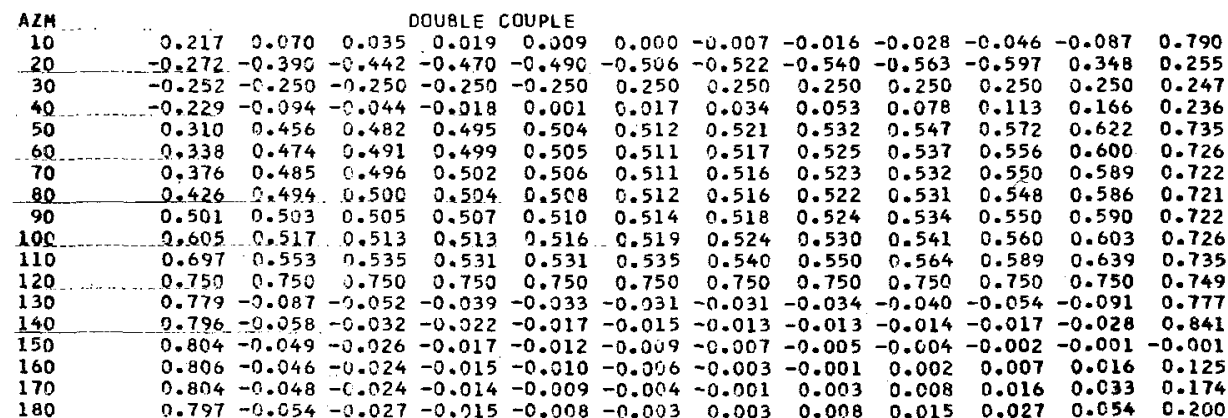


LEFT LATERAL REVERSE FAULT, DIP DIRECTION DIFFERENCE $=130.0$

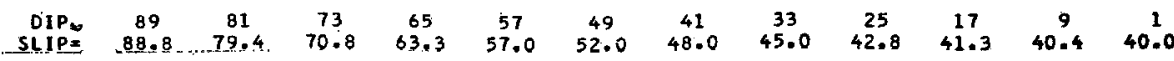

\begin{tabular}{|c|c|c|c|c|c|c|c|c|c|c|c|c|}
\hline $\begin{array}{l}A 2 H \\
10 \\
20 \\
30 \\
40 \\
40 \\
50 \\
60 \\
70 \\
80 \\
90 \\
100 \\
110 \\
120 \\
130 \\
140 \\
150 \\
160 \\
170 \\
180\end{array}$ & $\begin{array}{r}-0.251 \\
-0.252 \\
-0.254 \\
0.250 \\
0.250 \\
0.251 \\
0.251 \\
0.252 \\
0.253 \\
0.254 \\
0.254 \\
0.256 \\
-0.245 \\
-0.244 \\
-0.244 \\
-0.243 \\
-0.241 \\
-0.014\end{array}$ & $\begin{array}{r}-0.258 \\
-0.272 \\
-0.288 \\
0.252 \\
0.250 \\
0.255 \\
0.261 \\
0.268 \\
0.275 \\
0.281 \\
0.288 \\
0.298 \\
-0.269 \\
-0.203 \\
-0.206 \\
-0.196 \\
-0.188 \\
-0.108\end{array}$ & $\begin{array}{r}-0.274 \\
-0.299 \\
-0.325 \\
0.253 \\
0.250 \\
0.259 \\
0.271 \\
0.283 \\
0.294 \\
0.305 \\
0.315 \\
0.332 \\
-0.183 \\
-0.175 \\
-0.172 \\
-0.170 \\
-0.169 \\
-0.157\end{array}$ & $\begin{array}{l}-0.351 \\
-0.370 \\
0.253 \\
0.250 \\
0.264 \\
0.280 \\
0.296 \\
0.311 \\
0.323 \\
0.335 \\
0.356 \\
-0.170 \\
-0.161 \\
-0.160 \\
-0.162 \\
-0.169 \\
-0.182\end{array}$ & $\begin{array}{r}-0.508 \\
-0.433 \\
0.249 \\
0.250 \\
0.269 \\
0.289 \\
0.307 \\
0.323 \\
0.336 \\
0.347 \\
0.373 \\
-0.167 \\
-0.158 \\
-0.159 \\
-0.164 \\
-0.175 \\
-0.196\end{array}$ & $\begin{array}{r}\text { COUPLE } \\
-0.76 C \\
-0.678 \\
-0.549 \\
0.236 \\
0.250 \\
0.275 \\
0.298 \\
0.316 \\
0.331 \\
0.343 \\
0.354 \\
0.384 \\
-0.172 \\
-0.161 \\
-0.163 \\
-0.171 \\
-0.183 \\
-0.204\end{array}$ & $\begin{array}{r}-0.768 \\
-0.736 \\
0.289 \\
0.200 \\
0.249 \\
0.282 \\
0.305 \\
0.322 \\
0.335 \\
0.346 \\
0.356 \\
0.392 \\
-0.183 \\
-0.169 \\
-0.171 \\
-0.179 \\
-0.191 \\
-0.209\end{array}$ & $\begin{array}{r}-0.777 \\
-0.769 \\
0.188 \\
0.111 \\
0.249 \\
0.292 \\
0.312 \\
0.326 \\
0.337 \\
0.346 \\
0.356 \\
0.400 \\
-0.199 \\
-0.181 \\
-0.182 \\
-0.188 \\
-0.198 \\
-0.210\end{array}$ & $\begin{array}{r}-0.790 \\
-0.800 \\
0.128 \\
0.014 \\
0.249 \\
0.305 \\
0.320 \\
0.329 \\
0.337 \\
0.344 \\
0.355 \\
0.409 \\
-0.222 \\
-0.195 \\
-0.193 \\
-0.196 \\
-0.202 \\
-0.208\end{array}$ & $\begin{array}{r}-0.811 \\
-0.838 \\
0.083 \\
-0.019 \\
0.249 \\
0.327 \\
0.333 \\
0.336 \\
0.340 \\
0.346 \\
0.359 \\
0.425 \\
-0.256 \\
-0.211 \\
-0.203 \\
-0.201 \\
-0.200 \\
-0.198\end{array}$ & $\begin{array}{r}-0.856 \\
0.104 \\
0.043 \\
-0.017 \\
0.249 \\
0.373 \\
0.366 \\
0.362 \\
0.362 \\
0.367 \\
0.385 \\
0.452 \\
-0.313 \\
-0.229 \\
-0.206 \\
-0.194 \\
-0.182 \\
-0.168\end{array}$ & $\begin{array}{r}0.022 \\
0.013 \\
0.005 \\
-0.002 \\
0.250 \\
0.482 \\
0.478 \\
0.476 \\
0.475 \\
0.477 \\
0.482 \\
0.494 \\
0.532 \\
-0.248 \\
-0.091\end{array}$ \\
\hline $\begin{array}{l}A Z M \\
10 \\
20 \\
30 \\
40 \\
50 \\
60 \\
70 \\
80 \\
90 \\
100 \\
110 \\
120 \\
130 \\
140 \\
150 \\
160 \\
170 \\
180\end{array}$ & $\begin{array}{l}0.229 \\
0.246 \\
0.264 \\
5.280 \\
0.301 \\
0.326 \\
0.358 \\
0.456 \\
0.501 \\
0.645 \\
0.729 \\
0.765 \\
0.783 \\
0.792 \\
0.796 \\
0.795 \\
0.790 \\
0.782\end{array}$ & $\begin{array}{r}0.115 \\
0.180 \\
0.326 \\
0.415 \\
0.451 \\
0.469 \\
0.480 \\
0.490 \\
0.593 \\
0.525 \\
0.612 \\
0.861 \\
-.077 \\
-.061 \\
-0.057 \\
-.057 \\
-0.063 \\
-076\end{array}$ & $\begin{aligned} & D \\
& 0.056 \\
& 0.124 \\
& 0.385 \\
& 0.459 \\
& 0.478 \\
& 0.487 \\
& 0.493 \\
& 0.498 \\
& 0.505 \\
& 0.518 \\
& 0.574 \\
&-0.093 \\
&-6.044 \\
&-0.033 \\
&-6.030 \\
&-0.029 \\
&-0.032 \\
&-1.039\end{aligned}$ & $\begin{array}{c}\text { DOUELE } \\
0.031 \\
0.076 \\
0.435 \\
0.481 \\
0.491 \\
0.496 \\
0.499 \\
0.503 \\
0.597 \\
0.517 \\
0.561 \\
-0.072 \\
-0.0 .30 \\
-0.022 \\
-0.019 \\
-0.018 \\
-0.019 \\
-0.022\end{array}$ & $\begin{array}{r}\text { COUPLE } \\
0.015 \\
0.033 \\
0.478 \\
0.496 \\
0.500 \\
0.502 \\
0.504 \\
0.507 \\
0.510 \\
0.519 \\
0.558 \\
-0.062 \\
-0.024 \\
-0.016 \\
-0.013 \\
-0.011 \\
-0.011 \\
-0.012\end{array}$ & $\begin{array}{r}0.002 \\
-0.005 \\
0.516 \\
0.509 \\
0.508 \\
0.508 \\
0.509 \\
0.511 \\
0.514 \\
0.522 \\
0.560 \\
-0.058 \\
-0.020 \\
-0.012 \\
-0.008 \\
-0.006 \\
-0.005 \\
-0.004\end{array}$ & $\begin{array}{r}-0.010 \\
-0.042 \\
0.553 \\
0.522 \\
0.516 \\
0.514 \\
0.514 \\
0.515 \\
0.518 \\
0.526 \\
6.566 \\
-0.059 \\
-0.018 \\
-0.009 \\
-0.005 \\
-0.002 \\
0.000 \\
0.004\end{array}$ & $\begin{array}{r}-0.024 \\
-0.079 \\
0.589 \\
0.537 \\
0.526 \\
0.522 \\
0.521 \\
0.521 \\
0.524 \\
0.533 \\
0.577 \\
-0.064 \\
-0.017 \\
-0.007 \\
-0.002 \\
0.002 \\
0.006 \\
0.012\end{array}$ & $\begin{array}{r}-0 \\
0 \\
0 \\
0 \\
0 \\
0 \\
0 \\
0 \\
0 \\
0 \\
0 \\
-0 \\
-0 \\
-0\end{array}$ & $\begin{array}{r}-0.069 \\
0.843 \\
0.665 \\
0.588 \\
0.562 \\
0.551 \\
0.546 \\
0.546 \\
0.550 \\
0.564 \\
0.623 \\
-0.097 \\
-0.022 \\
-0.003 \\
0.007 \\
0.015 \\
0.024 \\
0.039\end{array}$ & $\begin{array}{r}-0.120 \\
0.801 \\
0.705 \\
0.641 \\
0.609 \\
0.592 \\
0.585 \\
0.584 \\
0.590 \\
0.609 \\
0.671 \\
0.856 \\
-0.036 \\
-0.002 \\
0.017 \\
0.032 \\
0.050 \\
0.076\end{array}$ & $\begin{array}{l}0.772 \\
0.762 \\
0.749 \\
0.737 \\
0.729 \\
0.723 \\
0.720 \\
0.720 \\
0.722 \\
0.728 \\
0.740 \\
0.764 \\
0.824 \\
0.001 \\
0.129 \\
0.174 \\
0.198 \\
0.215\end{array}$ \\
\hline
\end{tabular}

TABLE 9

LEFT LATERAL REVERSE FAUIT, DIP DIRECTION DIFFERENCE = 140.0

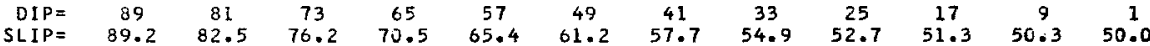

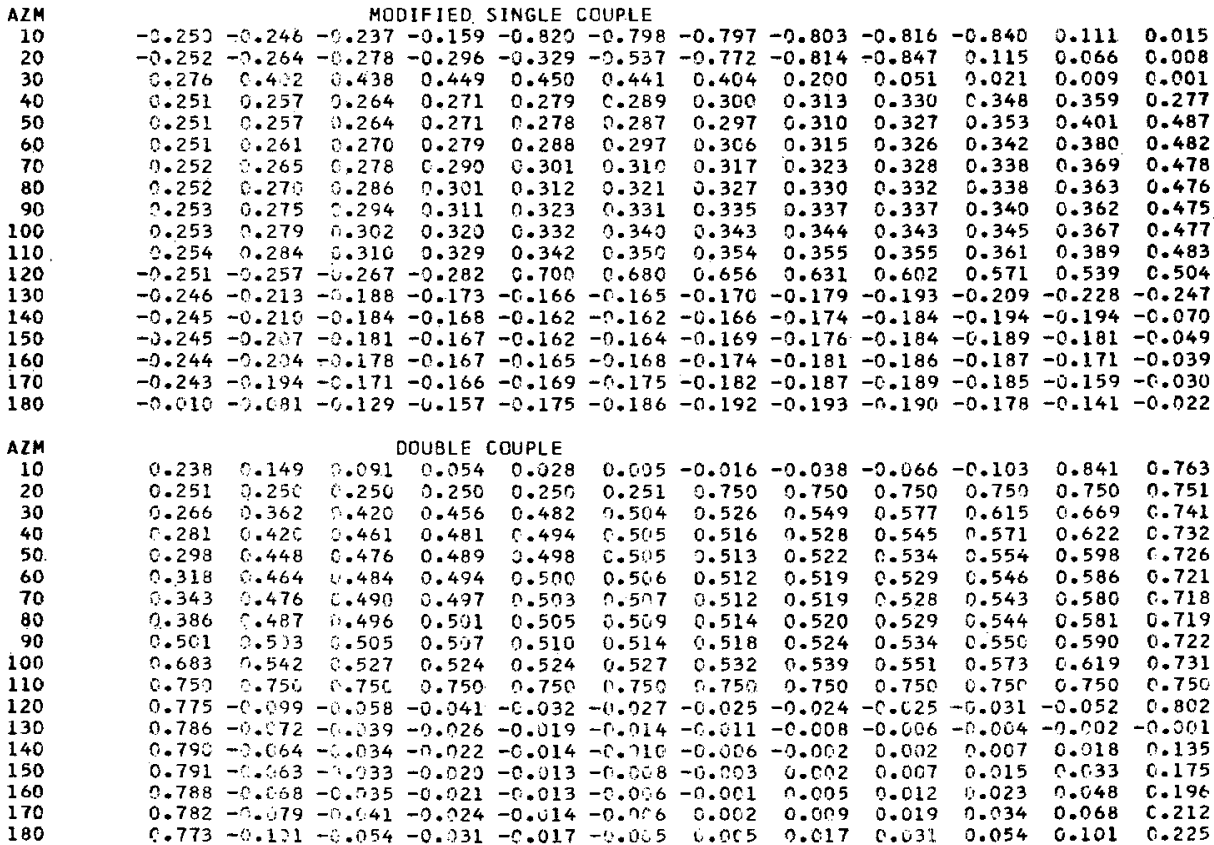

TABLE 10 
LEFT LATERAL REVERSE FAULT, DIP DIRECTION DIFFERENCE = 150.0

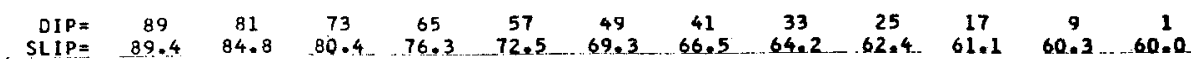

\begin{tabular}{|c|c|c|c|c|c|c|c|c|c|c|c|c|}
\hline $\begin{array}{l}A 2 M \\
10 \\
20 \\
30 \\
40 \\
50 \\
60 \\
70 \\
80 \\
90 \\
100 \\
110 \\
120 \\
130 \\
140 \\
150 \\
160 \\
170 \\
180\end{array}$ & $\begin{array}{r}-0.249 \\
-0.252 \\
0.253 \\
0.251 \\
0.251 \\
0.252 \\
0.252 \\
0.252 \\
0.253 \\
0.253 \\
0.254 \\
-0.247 \\
-0.246 \\
-0.246 \\
-0.246 \\
-0.245 \\
-0.244 \\
-0.006\end{array}$ & $\begin{array}{r}-0.240 \\
-0.270 \\
0.273 \\
0.262 \\
0.262 \\
0.265 \\
0.268 \\
0.271 \\
0.275 \\
0.278 \\
0.282 \\
-0.220 \\
-0.217 \\
-0.215 \\
-0.213 \\
-0.210 \\
-0.198 \\
-0.057\end{array}$ & $\begin{array}{r}M 0 D \\
-0.225 \\
-0.286 \\
0.294 \\
0.273 \\
0.273 \\
0.278 \\
0.283 \\
0.289 \\
0.294 \\
0.300 \\
0.307 \\
-0.198 \\
-0.193 \\
-0.191 \\
-0.188 \\
-0.184 \\
-0.173 \\
-0.097\end{array}$ & $\begin{array}{c}\text { DIFIED S } \\
-0.185 \\
-0.302 \\
0.315 \\
0.284 \\
0.284 \\
0.290 \\
0.297 \\
0.304 \\
0.311 \\
0.317 \\
0.326 \\
-0.183 \\
-0.177 \\
-0.174 \\
-0.173 \\
-0.170 \\
-0.163 \\
-0.126\end{array}$ & $\begin{array}{r}\text { SINGLE } \\
-0.045 \\
-0.316 \\
0.338 \\
0.297 \\
0.295 \\
0.301 \\
0.308 \\
0.316 \\
0.323 \\
0.330 \\
0.340 \\
-0.174 \\
-0.167 \\
-0.166 \\
-0.165 \\
-0.165 \\
-0.162 \\
-0.145\end{array}$ & $\begin{array}{l}\text { COUPLE } \\
-0.896 \\
-0.321 \\
0.362 \\
0.310 \\
0.306 \\
0.310 \\
0.317 \\
0.324 \\
0.331 \\
0.337 \\
0.349 \\
-0.171 \\
-0.164 \\
-0.163 \\
-0.164 \\
-0.165 \\
-0.165 \\
-0.159\end{array}$ & $\begin{array}{r}-0.858 \\
0.727 \\
0.389 \\
0.325 \\
0.317 \\
0.319 \\
0.324 \\
0.330 \\
0.335 \\
0.341 \\
0.355 \\
-0.17 .4 \\
-0.165 \\
-0.165 \\
-0.166 \\
-0.168 \\
-0.170 \\
-0.166\end{array}$ & $\begin{array}{r}-0.853 \\
0.008 \\
0.419 \\
0.343 \\
0.328 \\
0.326 \\
0.329 \\
0.333 \\
0.337 \\
0.342 \\
0.358 \\
-0.181 \\
-0.170 \\
-0.169 \\
-0.171 \\
-0.172 \\
-0.173 \\
-0.169\end{array}$ & $\begin{array}{r}-0.862 \\
0.042 \\
0.454 \\
0.365 \\
0.341 \\
0.334 \\
0.333 \\
0.334 \\
0.337 \\
0.342 \\
0.362 \\
-0.193 \\
-0.177 \\
-0.175 \\
-0.175 \\
-0.175 \\
-0.173 \\
-0.165\end{array}$ & $\begin{array}{r}-0.886 \\
0.034 \\
0.498 \\
0.393 \\
0.361 \\
0.347 \\
0.341 \\
0.339 \\
0.340 \\
0.345 \\
0.371 \\
-0.208 \\
-0.184 \\
-0.178 \\
-0.175 \\
-0.172 \\
-0.164 \\
-0.149\end{array}$ & $\begin{array}{r}0.072 \\
0.019 \\
0.568 \\
0.435 \\
0.401 \\
0.381 \\
0.369 \\
0.363 \\
0.362 \\
0.367 \\
0.402 \\
-0.227 \\
-0.178 \\
-0.166 \\
-0.157 \\
-0.147 \\
-0.131 \\
-0.108\end{array}$ & $\begin{array}{l}0.009 \\
0.002 \\
0.721 \\
0.492 \\
0.487 \\
0.482 \\
0.478 \\
0.476 \\
0.475 \\
0.477 \\
0.486 \\
0.247 \\
0.050 \\
0.038 \\
0.032 \\
-0.027 \\
0.021 \\
0.015\end{array}$ \\
\hline
\end{tabular}

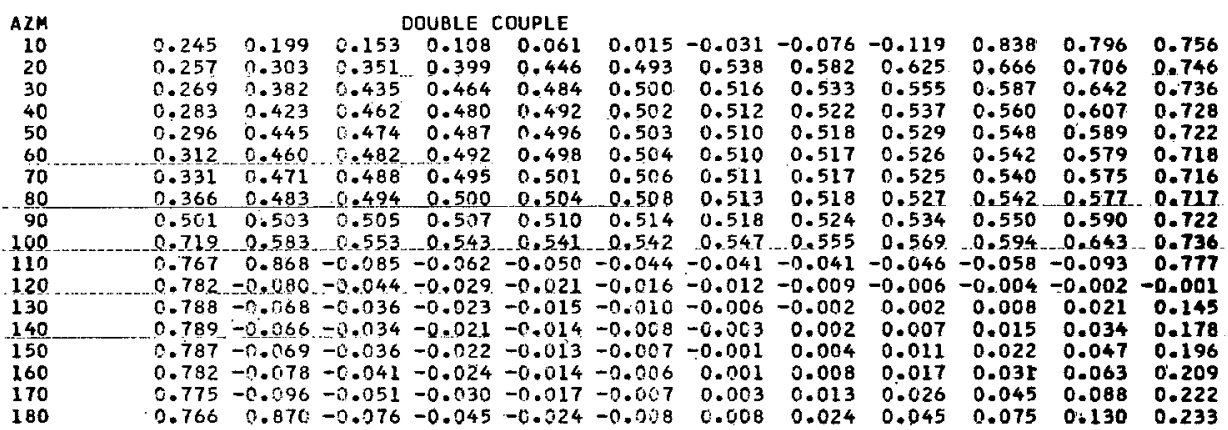
TABLE 11

LEFT LATERAL REVERSE FAULT, DIP DIRECTION DIFFERENCE $=160.0$

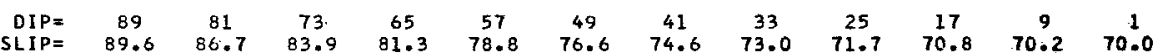

$A 2 M$
10
20
30
40
50
60
70
80
90
100
110
120
130
140
150
160
170
180

MODIFIED SINGLE COUPLE

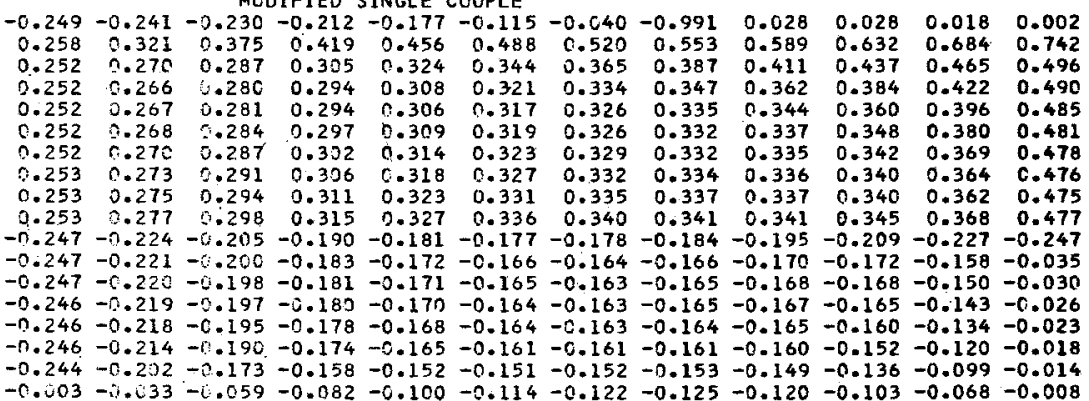

$A Z M$
10
20
30
40
50
60
70
80
90
100
110
120
130
140
150
160
170
180

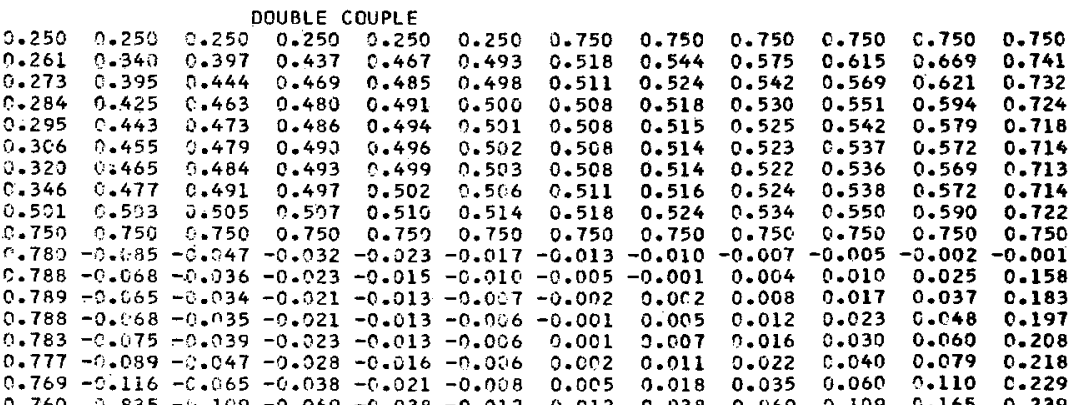

TABLE 12 
LEFT LATERAL REVERSE FAULT, DIP DIRECTION DIFFERENCE = 170.0

\begin{tabular}{|c|c|c|c|c|c|c|c|c|c|c|c|c|}
\hline $\begin{array}{r}D I P= \\
L L I P=\end{array}$ & $\begin{array}{c}89 \\
89.8\end{array}$ & $\begin{array}{c}81 \\
88.4\end{array}$ & $\begin{array}{c}73 \\
87.0\end{array}$ & $\begin{array}{c}65 \\
85.7\end{array}$ & $\begin{array}{c}57 \\
84.5\end{array}$ & $\begin{array}{c}49 \\
83.4\end{array}$ & $\begin{array}{c}41 \\
82.4\end{array}$ & $\begin{array}{c}33 \\
81.6\end{array}$ & $\begin{array}{c}25 \\
80.9\end{array}$ & $\begin{array}{c}17 \\
80.4\end{array}$ & 80.9 & 80.0 \\
\hline & $\begin{array}{r}0.304 \\
0.253 \\
0.252 \\
0.252 \\
0.252 \\
0.252 \\
0.253 \\
0.253 \\
0.253 \\
-0.247 \\
-0.247 \\
-0.247 \\
-0.247 \\
-0.247 \\
-0.247 \\
-0.246 \\
-0.245 \\
-0.030\end{array}$ & $\begin{array}{r}0.479 \\
0.280 \\
0.271 \\
0.276 \\
0.270 \\
0.271 \\
0.272 \\
10.274 \\
0.275 \\
-1.227 \\
-0.224 \\
-0.224 \\
-0.224 \\
-0.223 \\
-0.222 \\
-0.218 \\
-0.204 \\
-0.055\end{array}$ & $\begin{array}{r}M O D \\
6.528 \\
i .307 \\
0.290 \\
0.287 \\
0.287 \\
0.289 \\
6.291 \\
. .293 \\
6.294 \\
-0.209 \\
-6.204 \\
-.203 \\
-6.203 \\
-1.202 \\
-0.200 \\
-0.194 \\
-5.172 \\
-0.010\end{array}$ & $\begin{array}{l}\text { OIFIED } \\
0.563 \\
0.333 \\
0.308 \\
0.302 \\
0.302 \\
0.374 \\
0.306 \\
0.309 \\
0.311 \\
-0.195 \\
-0.187 \\
-0.187 \\
-0.186 \\
-0.186 \\
-0.183 \\
-0.176 \\
-0.150 \\
-0.014\end{array}$ & $\begin{array}{l}\text { SINGLE } \\
0.592 \\
0.358 \\
0.324 \\
0.315 \\
0.314 \\
0.316 \\
0.318 \\
0.321 \\
0.323 \\
-0.186 \\
-0.175 \\
-0.175 \\
-0.175 \\
-0.174 \\
-0.171 \\
-0.163 \\
-0.137 \\
-0.018\end{array}$ & $\begin{array}{c}\text { COUPLE } \\
0.618 \\
0.383 \\
0.339 \\
0.327 \\
0.324 \\
0.325 \\
0.327 \\
0.329 \\
0.331 \\
-0.181 \\
-0.168 \\
-0.167 \\
-0.167 \\
-0.166 \\
-0.164 \\
-0.155 \\
-0.128 \\
-0.022\end{array}$ & $\begin{array}{r}0.642 \\
0.407 \\
0.353 \\
0.337 \\
0.332 \\
0.331 \\
0.332 \\
0.334 \\
0.335 \\
-0.181 \\
-0.164 \\
-0.164 \\
-0.163 \\
-0.163 \\
-0.159 \\
-0.150 \\
-0.123 \\
-0.024\end{array}$ & $\begin{array}{r}0.665 \\
0.429 \\
0.366 \\
0.345 \\
0.338 \\
0.335 \\
0.335 \\
0.336 \\
0.337 \\
-0.186 \\
-0.164 \\
-0.163 \\
-0.162 \\
-0.161 \\
-0.157 \\
-0.146 \\
-0.118 \\
-0.025\end{array}$ & $\begin{array}{l}0.6 \\
0.4 \\
0.3 \\
0.3 \\
0.3 \\
0.3 \\
0.3 \\
0.3 \\
0.3 \\
-0.1 \\
-0.1 \\
-0.1 \\
-0.1 \\
-0.1 \\
-0.1 \\
-0.1 \\
-0.1 \\
-0.02\end{array}$ & $\begin{array}{r}0.707 \\
0.469 \\
0.403 \\
0.372 \\
0.356 \\
0.347 \\
0.343 \\
0.340 \\
0.340 \\
-0.210 \\
-0.163 \\
-0.161 \\
-0.158 \\
-0.153 \\
-0.143 \\
-0.126 \\
-0.093 \\
-0.019\end{array}$ & $\begin{array}{r}0.728 \\
0.485 \\
0.438 \\
0.408 \\
0.389 \\
0.376 \\
0.368 \\
0.364 \\
0.362 \\
-0.227 \\
-0.142 \\
-0.138 \\
-0.133 \\
-0.124 \\
-0.111 \\
-0.091 \\
-0.061 \\
-0.011\end{array}$ & $\begin{array}{r}0.747 \\
0.498 \\
0.492 \\
0.488 \\
0.484 \\
0.480 \\
0.478 \\
0.476 \\
0.475 \\
-0.246 \\
-0.027 \\
-0.025 \\
-0.023 \\
-0.020 \\
-0.016 \\
-0.012 \\
-0.007\end{array}$ \\
\hline & $\begin{array}{l}0.255 \\
0.266 \\
0.275 \\
0.285 \\
0.293 \\
0.302 \\
0.311 \\
0.326 \\
0.501 \\
0.778 \\
0.791 \\
0.793 \\
0.791 \\
0.787 \\
0.781 \\
0.773 \\
0.764 \\
0.755\end{array}$ & $\begin{array}{r}0.295 \\
0.365 \\
0.465 \\
0.427 \\
0.441 \\
0.451 \\
0.458 \\
0.468 \\
0.533 \\
-0.088 \\
-0.063 \\
-0.060 \\
-0.063 \\
-0.369 \\
-0.081 \\
-0.102 \\
0.861 \\
0.794\end{array}$ & $\begin{array}{r}0.337 \\
.421 \\
.450 \\
.463 \\
.471 \\
.476 \\
.480 \\
0.486 \\
0.505 \\
-0.250 \\
-0.033 \\
-5.331 \\
-0.032 \\
-.036 \\
-0.042 \\
-0.055 \\
-0.083 \\
-.835\end{array}$ & $\begin{array}{r}\text { OOUBLE } \\
0.381 \\
0.453 \\
0.471 \\
0.480 \\
0.484 \\
0.487 \\
0.490 \\
0.493 \\
0.507 \\
-0.033 \\
-0.020 \\
-0.019 \\
-0.019 \\
-0.921 \\
-0.025 \\
-0.932 \\
-0.050 \\
-0.121\end{array}$ & $\begin{array}{r}\text { COUPLE } \\
0.428 \\
0.475 \\
0.485 \\
0.492 \\
0.492 \\
0.494 \\
0.496 \\
0.499 \\
0.510 \\
-0.024 \\
-0.013 \\
-0.011 \\
-0.011 \\
-0.012 \\
-0.014 \\
-0.018 \\
-0.028 \\
-0.474\end{array}$ & $\begin{array}{r}0.478 \\
0.493 \\
0.497 \\
0.498 \\
0.499 \\
0.500 \\
0.501 \\
0.503 \\
0.514 \\
-0.018 \\
-0.008 \\
-0.006 \\
-0.005 \\
-0.005 \\
-0.0155 \\
-0.046 \\
-0.010 \\
-0.025\end{array}$ & $\begin{array}{r}0.528 \\
0.510 \\
0.507 \\
0.506 \\
0.505 \\
0.505 \\
0.506 \\
0.508 \\
0.518 \\
-0.014 \\
-0.003 \\
-0.001 \\
0.000 \\
0.001 \\
0.002 \\
0.004 \\
0.008 \\
0.025\end{array}$ & $\begin{array}{r}0.577 \\
0.529 \\
0.518 \\
0.514 \\
0.512 \\
0.511 \\
0.511 \\
0.513 \\
0.524 \\
-0.010 \\
0.002 \\
0.004 \\
0.006 \\
0.008 \\
0.011 \\
0.015 \\
0.026 \\
0.074\end{array}$ & $\begin{array}{r}0.623 \\
0.552 \\
0.533 \\
0.525 \\
0.521 \\
0.519 \\
0.519 \\
0.521 \\
0.534 \\
-0.007 \\
0.007 \\
0.010 \\
0.013 \\
0.016 \\
0.021 \\
0.029 \\
0.048 \\
0.121\end{array}$ & $\begin{array}{r}0.666 \\
0.585 \\
0.556 \\
0.543 \\
0.536 \\
0.533 \\
0.531 \\
0.533 \\
0.550 \\
-0.005 \\
0.015 \\
0.020 \\
0.024 \\
0.029 \\
0.037 \\
0.051 \\
0.081 \\
0.165\end{array}$ & $\begin{array}{r}0.706 \\
0.640 \\
0.603 \\
0.582 \\
0.570 \\
0.564 \\
0.561 \\
0.564 \\
0.590 \\
-0.003 \\
0.033 \\
0.042 \\
0.050 \\
0.060 \\
0.074 \\
0.097 \\
0.136 \\
0.206\end{array}$ & $\begin{array}{l}0.720 \\
0.714 \\
0.710 \\
0.708 \\
0.710 \\
0.722 \\
-0.001 \\
0.175 \\
0.190 \\
0.199 \\
0.207 \\
0.216 \\
0.225 \\
0.235 \\
0.245\end{array}$ \\
\hline
\end{tabular}

TABLE 13

LEFT LATERAL REVERSE FAULT, DIP DIRECTION CIFFERENCE $=180.0$

\begin{tabular}{|c|c|c|c|c|c|c|c|c|c|c|c|c|}
\hline $\begin{array}{r}\text { OIP }= \\
\text { SLIP }=\end{array}$ & $\begin{array}{c}89 \\
90.0\end{array}$ & $\begin{array}{c}8 i \\
93 . j\end{array}$ & $\begin{array}{c}73 \\
90.6\end{array}$ & $\begin{array}{c}6.5 \\
90.0\end{array}$ & $\begin{array}{c}57 \\
90.0\end{array}$ & $\begin{array}{c}49 \\
90.5\end{array}$ & $\begin{array}{c}41 \\
90.0\end{array}$ & $\begin{array}{c}33 \\
90.0\end{array}$ & $\begin{array}{r}25 \\
90.0\end{array}$ & $\begin{array}{c}17 \\
90.0\end{array}$ & $9 \stackrel{9}{90.0}$ & $\stackrel{1}{90.0}$ \\
\hline & $\begin{array}{r}0.256 \\
0.253 \\
0.253 \\
0.253 \\
0.253 \\
0.253 \\
0.253 \\
0.253 \\
-0.247 \\
-0.247 \\
-0.247 \\
-0.247 \\
-0.247 \\
-0.247 \\
-0.247 \\
-0.247 \\
-0.244 \\
0.289\end{array}$ & $\begin{array}{r}0.299 \\
0.279 \\
0.274 \\
.273 \\
0.273 \\
0.274 \\
.274 \\
0.275 \\
-0.225 \\
-0.225 \\
-.0 .226 \\
-0.226 \\
-0.227 \\
-0.227 \\
-0.226 \\
-0.221 \\
-0.231 \\
0.251\end{array}$ & 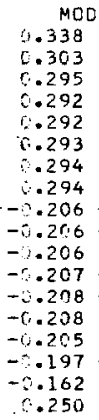 & $\begin{array}{l}\text { IFIED S } \\
0.371 \\
0.324 \\
0.312 \\
0.308 \\
0.378 \\
0.379 \\
0.310 \\
0.310 \\
-0.189 \\
-0.190 \\
-0.190 \\
-0.191 \\
-0.192 \\
-0.192 \\
-0.188 \\
-0.176 \\
-0.129 \\
0.250\end{array}$ & $\begin{array}{r}\text { INGLE } \\
0.399 \\
0.342 \\
0.326 \\
0.321 \\
0.320 \\
C .321 \\
0.322 \\
0.323 \\
-0.177 \\
-0.177 \\
-0.178 \\
-0.179 \\
-C .180 \\
-0.179 \\
-0.174 \\
-0.158 \\
-C .101 \\
0.250\end{array}$ & $\begin{array}{c}\text { OUPLE } \\
0.422 \\
0.356 \\
0.337 \\
0.331 \\
0.329 \\
0.329 \\
0.339 \\
0.331 \\
-0.169 \\
-0.169 \\
-0.170 \\
-0.171 \\
-0.171 \\
-0.169 \\
-0.163 \\
-0.144 \\
-0.078 \\
0.250\end{array}$ & $\begin{array}{r}0.441 \\
0.368 \\
0.346 \\
0.338 \\
0.335 \\
0.335 \\
0.335 \\
0.335 \\
-0.165 \\
-0.165 \\
-0.165 \\
-0.165 \\
-0.165 \\
-0.162 \\
-0.154 \\
-0.132 \\
-0.059 \\
0.250\end{array}$ & $\begin{array}{r}0.457 \\
0.379 \\
0.353 \\
0.343 \\
0.339 \\
0.337 \\
0.337 \\
0.337 \\
-0.163 \\
-0.163 \\
-0.163 \\
-0.163 \\
-0.161 \\
-0.157 \\
-0.147 \\
-0.121 \\
-0.043 \\
0.250\end{array}$ & $\begin{array}{r}0.471 \\
0.392 \\
0.362 \\
0.348 \\
0.342 \\
0.339 \\
0.338 \\
0.337 \\
-0.163 \\
-0.163 \\
-0.162 \\
-0.161 \\
-0.158 \\
-0.152 \\
-0.138 \\
-0.108 \\
-0.029 \\
0.250\end{array}$ & $\begin{array}{r}0.483 \\
0.411 \\
0.377 \\
0.360 \\
0.350 \\
0.345 \\
0.342 \\
0.340 \\
-0.160 \\
-0.160 \\
-0.158 \\
-0.155 \\
-0.150 \\
-0.140 \\
-0.123 \\
-0.089 \\
-0.017 \\
0.250\end{array}$ & $\begin{array}{r}0.492 \\
0.443 \\
0.413 \\
0.393 \\
0.380 \\
0.371 \\
0.366 \\
0.363 \\
-0.138 \\
-0.137 \\
-0.134 \\
-0.129 \\
-0.120 \\
-0.107 \\
-0.087 \\
-0.057 \\
-0.008 \\
0.250\end{array}$ & $\begin{array}{r}0.499 \\
0.493 \\
0.488 \\
0.484 \\
0.481 \\
0.478 \\
0.477 \\
0.475 \\
-0.025 \\
-0.025 \\
-0.023 \\
-0.022 \\
-0.0119 \\
-0.016 \\
-0.012 \\
-0.007 \\
-0.001 \\
0.251\end{array}$ \\
\hline & $\begin{array}{l}0.260 \\
0.269 \\
0.278 \\
0.286 \\
0.292 \\
0.297 \\
0.321 \\
0.303 \\
0.007 \\
0.803 \\
0.801 \\
0.797 \\
0.792 \\
0.786 \\
0.778 \\
0.769 \\
0.765 \\
2.751\end{array}$ & $\begin{array}{r}0.332 \\
0.383 \\
0.412 \\
0.429 \\
0.439 \\
0.446 \\
0.456 \\
0.452 \\
-1.0013 \\
-0.048 \\
-1.050 \\
-7.054 \\
-0.061 \\
-10.071 \\
-0.688 \\
-0.117 \\
0.832 \\
-.750\end{array}$ & $\begin{array}{r}. .388 \\
.435 \\
.454 \\
.464 \\
0.470 \\
6.473 \\
0.475 \\
-.476 \\
-.000 \\
-.024 \\
-.025 \\
-.027 \\
-.030 \\
-0.036 \\
-.044 \\
-.065 \\
-.112 \\
\therefore .750\end{array}$ & $\begin{array}{r}0.428 \\
0.462 \\
0.474 \\
0.479 \\
0.483 \\
0.485 \\
0.486 \\
0.486 \\
0.003 \\
-0.014 \\
-0.014 \\
-0.015 \\
-0.017 \\
-0.021 \\
-0.026 \\
-0.038 \\
-0.072 \\
0.750\end{array}$ & $\begin{array}{r}\text { OUPLE } \\
0.460 \\
0.479 \\
0.486 \\
0.489 \\
0.491 \\
0.492 \\
0.492 \\
0.493 \\
0.066 \\
-0.007 \\
-0.008 \\
-0.008 \\
-0.009 \\
-0.011 \\
-0.014 \\
-0.021 \\
-0.040 \\
0.750\end{array}$ & $\begin{array}{r}0.487 \\
0.493 \\
0.496 \\
0.497 \\
0.497 \\
0.497 \\
0.498 \\
0.498 \\
0.009 \\
-0.002 \\
-0.062 \\
-0.043 \\
-0.263 \\
-0.063 \\
-0.064 \\
-0.027 \\
-0.213 \\
0.750\end{array}$ & $\begin{array}{l}0.513 \\
0.507 \\
0.504 \\
0.503 \\
0.503 \\
0.503 \\
0.502 \\
0.502 \\
0.014 \\
0.002 \\
0.002 \\
0.003 \\
0.003 \\
0.003 \\
0.004 \\
0.007 \\
0.013 \\
0.250\end{array}$ & $\begin{array}{l}0.540 \\
0.521 \\
0.514 \\
0.511 \\
0.509 \\
0.508 \\
0.508 \\
0.507 \\
0.019 \\
0.007 \\
0.008 \\
0.008 \\
0.009 \\
0.011 \\
0.014 \\
0.021 \\
0.040 \\
0.250\end{array}$ & $\begin{array}{l}0.572 \\
0.538 \\
0.526 \\
0.521 \\
0.517 \\
0.515 \\
0.514 \\
0.514 \\
0.027 \\
0.014 \\
0.014 \\
0.015 \\
0.017 \\
0.021 \\
0.026 \\
0.038 \\
0.072 \\
0.250\end{array}$ & $\begin{array}{l}0.612 \\
0.565 \\
0.546 \\
0.536 \\
0.530 \\
0.527 \\
0.525 \\
0.524 \\
0.042 \\
0.024 \\
0.025 \\
0.027 \\
0.030 \\
0.036 \\
0.046 \\
0.065 \\
0.112 \\
0.250\end{array}$ & $\begin{array}{l}0.668 \\
0.617 \\
0.588 \\
0.571 \\
0.561 \\
0.554 \\
0.550 \\
0.548 \\
0.077 \\
0.048 \\
0.050 \\
0.054 \\
0.061 \\
0.071 \\
0.088 \\
0.117 \\
0.168 \\
0.250\end{array}$ & $\begin{array}{l}0.740 \\
0.731 \\
0.722 \\
0.714 \\
0.708 \\
0.703 \\
0.699 \\
0.697 \\
0.219 \\
0.197 \\
0.199 \\
0.203 \\
0.208 \\
0.214 \\
0.222 \\
0.231 \\
0.240 \\
0.250\end{array}$ \\
\hline
\end{tabular}

TABLE 14 
from the strike is $62^{\circ}$. We find in table 9 the value of $\phi_{R}-\phi_{L}$ for the above parameters of $17^{\circ}, 130^{\circ}$ and $60^{\circ}$ respectively. The value is 0.327 for the modified single couple and 0.551 for the double couple. Values corresponding more precisely to the given parameters may be obtained by interpolation.

If plane $b$ is the actual fault, the motion is left-lateral normal, the dip angle is $78^{\circ}$, and the azimuth to Pasadena from the strike is $194^{\circ}$. According to the relations described in the Introduction, the value of $\phi_{R}-\phi_{L}$ for left-lateral normal motion at an azimuth $194^{\circ}$ differs by 0.5 circle from that at $-14^{\circ}\left(=346^{\circ}\right)$ for right-lateral normal motion. The value corresponding to right-lateral normal motion is the same as that corresponding to left-lateral reverse motion for which the table gives the
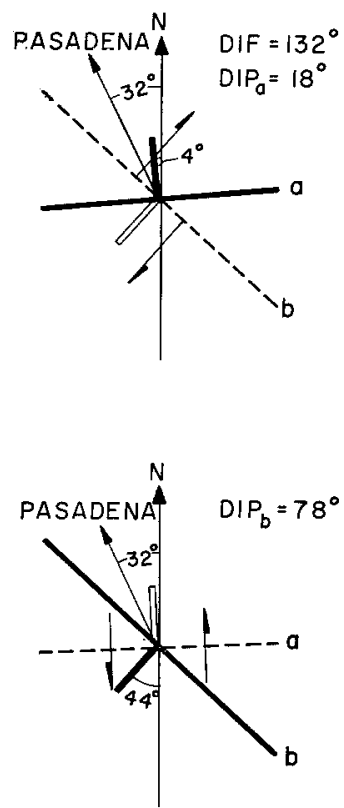

Frg. 2. Fault plane solutions of shock M3 of Part 2. DIF indicates the difference in dip direction between plane $a$ and $b$. DIP indicates the dip angle of each plane. The horizontal projection of the motion direction is indicated by pairs of arrows.

value. Therefore, the value of $\phi_{R}-\phi_{L}$ for the given fault motion at an azimuth $194^{\circ}$ differs by 0.50 from that at $346^{\circ}$ for left-lateral reverse motion. The latter value may be obtained according to the conjugate relation described before from the value at an azimuth of $166^{\circ}$ (= $=346-180$ ). Taking the dip direction difference $130^{\circ}$, we get from table 9 the value of $\phi_{R}-\phi_{L}$ corresponding to dip angle of $78^{\circ}$ and az.muth of $166^{\circ}$ as -0.183 for the modified single couple and -0.049 for the double couple by interpolation.

Then, the conjugate relation gives the corresponding values at $346^{\circ}$ as 0.183 and 0.049 . Finally, the values for the given fault motion are obtained as 0.683 and 0.549 by adding 0.50 to the above values. The value corresponding to the double couple model should be the same for plane $a$ and plane $b$. The values obtained are 0.551 and 0.549 , and show agreement within the error of interpolation. 


\section{ACKNOWLEDGMENT}

Drs. Frank Press and Dr. Clarence R. Allen kindly read through the manuseript and gave the author valuable suggestions and advice.

The present research was supported by Grant No. AF-AFOSR-25-63 of the Air Force Office of Scientific Research as part of the Advanced Research Projects Agency Project veLA.

\section{REFERENCES}

Aki, K.

1963. "Study of Love and Rayleigh waves from earthquakes with fault plane solutions or with known faulting. Part 1. A phase difference method based on a new model of earthquake source," Bull. Seism. Soc. Am. 54, 511-528. "Part 2. Application of the phase difference method," Bull. Seism. Soc. Am. 54, 529-558.

Haskell, N. A.

1963. "Radiation pattern of Rayleigh waves from a fault of arbitrary dip and direction of motion in a homogeneous medium," Bull. Seism. Soc. Am., 53, 619-642.

Seismological Laboratory

California Institute of Techiology

Pasadena, Califoria

Division of The Geological Sciences, Contribution No. 1214

Manuscript received November 21, 1963 